\title{
On finite $W$-algebras for Lie algebras and superalgebras
}

\author{
Elena Poletaeva \\ Department of Mathematics, University of Texas-Pan American, Edinburg, TX 78539 \\ E-mail address: elenap@utpa.edu
}

\begin{abstract}
The finite $W$-algebras are certain associative algebras associated to a complex semi-simple or reductive Lie algebra $\mathfrak{g}$ and a nilpotent element $e$ of $\mathfrak{g}$. Due to recent results of I. Losev, A. Premet and others, finite $W$-algebras play a very important role in description of primitive ideals. In the full generality, the finite $W$-algebras were introduced by A. Premet. It is a result of B. Kostant that for a regular nilpotent (principal) element $e$, the finite $W$-algebra coincides with the center of $U(\mathfrak{g})$. Premet's definition makes sense for a simple Lie superalgebra $\mathfrak{g}=\mathfrak{g}_{\overline{0}} \oplus \mathfrak{g}_{\overline{1}}$ in the case when $\mathfrak{g}_{\overline{0}}$ is reductive, $\mathfrak{g}$ admits an invariant super-symmetric bilinear form, and $e$ is an even nilpotent element. We show that certain results of A. Premet can be generalized for classical Lie superalgebras. We consider the case when $e$ is an even regular nilpotent element. The associated finite $W$-algebra is called principal. Kostant's result does not hold in this case. This is joint work with V. Serganova.
\end{abstract}

\section{Introduction}

A finite $W$-algebra is certain associative algebra associated to a complex semisimple Lie algebra $\mathfrak{g}$ and a nilpotent element $e \in \mathfrak{g}$. It is a generalization of the universal enveloping algebra $U(\mathfrak{g})$.

The finite $W$-algebras are quantizations of Poisson algebras of functions on the Slodowy (i.e. transversal) slice at $e$ to the adjoint orbit $A d(G) e$, where $\mathfrak{g}=\operatorname{Lie}(G)$ [Pr1]. Due to recent results of I. Losev, A. Premet and others, finite $W$-algebras play a very important role in description of primitive ideals [L2, L3, $\operatorname{Pr} 2, \operatorname{Pr} 3$ ].

The key ideas for finite $W$-algebras appeared in the study of classical and quantum affine $W$-algebras [B, F1, F2]. In the full generality, the finite $W$-algebras for semi-simple Lie algebras were introduced by A. Premet [Pr1]. The construction of finite $W$-algebras is based on the study of Whittaker vectors and Whittaker modules in the famous work of B. Konstant [Ko].

This survey was presented during the 3rd Graduate Research Conference in Algebra and Representation Theory at Kansas State University. 
Finite $W$-algebras for Lie algebras have been extensively studied by I. Losev, V. Ginzburg, W. L. Gan, J. Brundan, S. Goodwin, A. Kleshchev, W. Wang and other mathematicians and physicists [L1, BG, GG, BK1, BK2, W].

In Section 2 we discuss Dynkin and good Z-gradings of $\mathfrak{g}$. In Sections 3-5 we review various equivalent definitions of finite $W$-algebra for $\mathfrak{g}$. In Section 6 we describe Kazhdan filtration on finite $W$-algebras. In Sections 7 and 8 we give examples of the principal finite $W$-algebra for $\mathfrak{g}=\mathfrak{g l}(2)$ and $\mathfrak{g l}(n)$.

In Sections 9-11 we recall the definition of a Lie superalgebra, main examples and classification of simple finite-dimensional Lie superalgebras. In Section 12 we review the definition of finite $W$-algebras for Lie superalgebras. In Section 13 we recall the notion of defect of a basic Lie superalgebra. In section 14 we describe the principal finite $W$-algebras for Lie superalgebras of type I and defect one. In Section 15 we outline the case when $\mathfrak{g}=\mathfrak{g l}(n \mid n)$. J.Brown, J.Brundan and $\mathrm{S}$. Goodwin have recently described the principal finite $W$-algebra of the Lie superalgebra $\mathfrak{g l}(m \mid n)$ as a certain truncation of a shifted version of the super-Yangian of $\mathfrak{g l}(1 \mid 1)$. In Section 16 we review the notion the super-Yangian of $\mathfrak{g l}(m \mid n)$. In sections 17 and 18 we consider the case when $\mathfrak{g}=Q(n)$. We describe the principal finite $W$-algebra for $Q(n)$ in terms of generators and relations, and show that it is isomorphic to a factor algebra of the super-Yangian of $Q(1)$. In Section 19 we describe the principal finite $W$-algebras for the family of simple exceptional Lie superalgebras $\Gamma\left(\sigma_{1}, \sigma_{2}, \sigma_{3}\right)$. In Sections 20 and 21 we describe the principal finite $W$-algebra for $\mathfrak{v s p}(1 \mid 2)$ and obtain partial results and formulate a conjecture for $\mathfrak{v} \mathfrak{s p}(1 \mid 2 n)$.

\section{Preliminaries}

Let $\mathfrak{g}$ be a finite-dimensional semi-simple or reductive Lie algebra over $\mathbb{C}$ and $(\cdot \mid \cdot)$ be a non-degenerate invariant symmetric bilinear form on $\mathfrak{g}$.

Definition 2.1. A bilinear form $(\cdot \mid \cdot)$ on $\mathfrak{g}$ is $\mathfrak{g}$-invariant if

$$
([x, y] \mid z)=(x \mid[y, z]) \quad \text { for all } x, y, z \in \mathfrak{g} .
$$

Definition 2.2. Adjoint representation of $\mathfrak{g}$.

For any $x \in \mathfrak{g}, \operatorname{ad}(x)$ is the endomorphism of $\mathfrak{g}$ defined as follows:

$$
a d(x)(y)=[x, y] \text { for all } y \in \mathfrak{g} .
$$

Definition 2.3. An element $e \in \mathfrak{g}$ is called nilpotent if ad $(e)$ is a nilpotent endomorphism of $\mathfrak{g}$.

Example 2.1. $\mathfrak{g}=\mathfrak{g l}(n),(a \mid b)=\operatorname{tr}(a b)$.

$e \in \mathfrak{g l}(n)$ is nilpotent if and only if $e$ is an $n \times n$-matrix with eigenvalues zero. 
Definition 2.4. A nilpotent element $e \in \mathfrak{g}$ is regular nilpotent if $\mathfrak{g}^{e}:=\operatorname{Ker}(\operatorname{ad}(e))$ attains the minimal dimension, which is equal to the rank of $\mathfrak{g}$.

Example 2.2. $\mathfrak{g}=\mathfrak{g l}(n)$.

$$
\begin{gathered}
e=J_{n}=\left(\begin{array}{cccccc}
0 & 1 & 0 & 0 & 0 & 0 \\
0 & 0 & 1 & 0 & 0 & 0 \\
0 & 0 & 0 & \cdots & 0 & 0 \\
0 & 0 & 0 & 0 & 1 & 0 \\
0 & 0 & 0 & 0 & 0 & 1 \\
0 & 0 & 0 & 0 & 0 & 0
\end{array}\right) \quad \text { is a regular nilpotent element. } \\
\mathfrak{g}^{e}=<e, e^{2}, \ldots, e^{n-1}, z>, \quad z=<1_{n}>, \quad \operatorname{dim}^{e}=n .
\end{gathered}
$$

Theorem 2.1. Jacobson-Morozov [C].

Associated to a nonzero nilpotent element $e \in \mathfrak{g}$, there always exists an $\mathfrak{s l}(2)$-triple $\{e, h, f\}$ which satisfies

$$
[e, f]=h, \quad[h, e]=2 e, \quad[h, f]=-2 f .
$$

Proof. Induction on dim $\mathfrak{g}$.

Example 2.3. $\mathfrak{g}=\mathfrak{g l}(n), e=J_{n}$. Let $h=\operatorname{diag}(n-1, n-3, \ldots, 3-n, 1-n)$ and

$$
f=\left(\begin{array}{cccccc}
0 & 0 & 0 & 0 & 0 & 0 \\
a_{1} & 0 & 0 & 0 & 0 & 0 \\
0 & a_{2} & 0 & \cdots & 0 & 0 \\
0 & 0 & \ldots & 0 & 0 & 0 \\
0 & 0 & 0 & \ldots & 0 & 0 \\
0 & 0 & 0 & 0 & a_{n-1} & 0
\end{array}\right) \text {, where } a_{i}=i(n-i) \text { for } 1 \leq i \leq n-1
$$

Then $e, h$ and $f$ span an $\mathfrak{s l}(2)$.

\section{3. $\mathbb{Z}$-gradings}

Definition 3.1. A Lie algebra $\mathfrak{g}$ is $\mathbb{Z}$-graded if

$$
\mathfrak{g}=\oplus_{j \in \mathbb{Z}} \mathfrak{g}_{j}, \quad\left[\mathfrak{g}_{j}, \mathfrak{g}_{k}\right] \subseteq \mathfrak{g}_{j+k} .
$$

Definition 3.2. A Dynkin $\mathbb{Z}$-grading.

Let $\mathfrak{s l}(2)=<e, h, f>$. The eigenspace decomposition of the adjoint action

$$
\operatorname{ad}(h): \mathfrak{g} \longrightarrow \mathfrak{g}
$$

provides a $\mathbb{Z}$-grading: $\mathfrak{g}=\oplus_{j \in \mathbb{Z}} \mathfrak{g}_{j}$, where $\mathfrak{g}_{j}=\{x \in \mathfrak{g} \mid \operatorname{ad}(h)(x)=j x\}$. 
A Dynkin $\mathbb{Z}$-grading satisfies the following properties:

(1) $e \in \mathfrak{g}_{2}$,

(2) $\operatorname{ad}(e): \mathfrak{g}_{j} \longrightarrow \mathfrak{g}_{j+2}$ is injective for $j \leq-1$,

(3) $\operatorname{ad}(e): \mathfrak{g}_{j} \longrightarrow \mathfrak{g}_{j+2}$ is surjective for $j \geq-1$,

(4) $\mathfrak{g}^{e} \subset \oplus_{j \geq 0} \mathfrak{g}_{j}$,

(5) $\left(\mathfrak{g}_{i} \mid \mathfrak{g}_{j}\right)=0$ unless $i+j=0$,

(6) $\operatorname{dim} \mathfrak{g}^{e}=\operatorname{dim} \mathfrak{g}_{0}+\operatorname{dim} \mathfrak{g}_{1}$.

Proof. (1) follows from the definition of $s \mathrm{l}(2),(2)$ and (3) follow from the theory of finite-dimensional irreducible $\mathfrak{s l}(2)$-modules, (4), (5) and (6) are easy to prove. We will show that these properties are valid for more general type of $\mathbb{Z}$-gradings, called good $\mathbb{Z}$-gradings.

Definition 3.3. A good $\mathbb{Z}$-grading.

$A \mathbb{Z}$-grading $\mathfrak{g}=\oplus_{j \in \mathbb{Z}} \mathfrak{g}_{j}$ for a semi-simple Lie algebra $\mathfrak{g}$ is good for , if it satisfies the conditions (1)-(3).

For a reductive $\mathfrak{g}$, there is an additional condition: the center of $\mathfrak{g}$ is in $\mathfrak{g}_{0}$. Note that good $\mathbb{Z}$-gradings of simple finite-dimensional Lie algebras over an algebraically closed field of characteristic zero were classified in [EK].

Proposition 3.1. Properties (4)-(6) remain to be valid for every good $\mathbb{Z}$-grading of $\mathfrak{g}$.

Proof. (see $[\mathrm{W}]$ )

(4) $\mathfrak{g}^{e}$ is a $\mathbb{Z}$-graded Lie subalgebra of $\mathfrak{g}$, and (4) follows from (2).

(5) For any $\mathbb{Z}$-grading $\mathfrak{g}=\oplus_{j \in \mathbb{Z}} \mathfrak{g}_{j}$ there exists a semi-simple element $h \in[\mathfrak{g}, \mathfrak{g}]$ such that

$$
\mathfrak{g}_{j}=\{x \in \mathfrak{g} \mid[h, x]=j x\} .
$$

Let $\partial: \mathfrak{g} \longrightarrow \mathfrak{g}$ be the degree operator:

$$
\partial(x):=j x \text { for } x \in \mathfrak{g}_{j},
$$

then $\partial$ is a derivation of the semi-simple Lie algebra $[\mathfrak{g}, \mathfrak{g}]$, hence $\partial$ is an inner derivation of $[\mathfrak{g}, \mathfrak{g}]$, given by $a d(h)$ for some semi-simple element $h \in[\mathfrak{g}, \mathfrak{g}]$. Then $\partial=a d(h)$ as derivations of $[\mathfrak{g}, \mathfrak{g}] \oplus \operatorname{center}(\mathfrak{g})$, since the equality hold on the center $(\mathfrak{g})$ too. For $x \in \mathfrak{g}_{i}, y \in \mathfrak{g}_{j}$, we have

$$
-i(x \mid y)=([x, h] \mid y)=(x \mid[h, y])=j(x \mid y) .
$$

Then (5) holds.

(6) From (2) and (3),

$$
\operatorname{ad}(e): \mathfrak{g}_{-1} \longrightarrow \mathfrak{g}_{1} \text { is a bijection. }
$$


An exact sequence of vector spaces

$$
0 \longrightarrow \mathfrak{g}^{e} \longrightarrow \mathfrak{g}_{-1} \oplus \mathfrak{g}_{0} \oplus\left(\oplus_{j>0} \mathfrak{g}_{j}\right) \stackrel{\operatorname{ad}(e)}{\longrightarrow} \oplus_{j>0} \mathfrak{g}_{j} \longrightarrow 0
$$

is well-defined by (2) and (3). Then (6) holds.

Example 3.1. Associated to $e=0$, we have a good $\mathbb{Z}$-grading with $\mathfrak{g}_{0}=\mathfrak{g}$.

Exercise 1. Properties (2) and (3) are equivalent for any $\mathbb{Z}$-grading $\mathfrak{g}=\oplus_{j \in \mathbb{Z}} \mathfrak{g}_{j}$.

Remark 3.1. Obviously, if a $\mathbb{Z}$-grading is Dynkin, then it is a good $\mathbb{Z}$-grading. However, not every good $\mathbb{Z}$-grading is Dynkin.

Example 3.2. $\mathfrak{g}=\mathfrak{g l}(3)$

$$
e=E_{13}=\left(\begin{array}{ccc}
0 & 0 & 1 \\
0 & 0 & 0 \\
0 & 0 & 0
\end{array}\right), \quad h=\left(\begin{array}{ccc}
1 & 0 & 0 \\
0 & 0 & 0 \\
0 & 0 & -1
\end{array}\right), \quad f=E_{31}=\left(\begin{array}{ccc}
0 & 0 & 0 \\
0 & 0 & 0 \\
1 & 0 & 0
\end{array}\right) .
$$

Note that $e$ is a non-regular nilpotent element, $\mathfrak{s l}(2)=<e, h, f>$ defines a Dynkin $\mathbb{Z}$-grading of $\mathfrak{g l}(3)$, whose degrees on the elementary matrices $E_{i j}$ are

$$
\begin{gathered}
\left(\begin{array}{ccc}
0 & 1 & 2 \\
-1 & 0 & 1 \\
-2 & -1 & 0
\end{array}\right) . \\
\operatorname{dim}\left(\mathfrak{g}^{e}\right)=\operatorname{dim} \mathfrak{g}_{0}+\operatorname{dim} \mathfrak{g}_{1}=5, \quad \mathfrak{g}^{e}=<E_{11}+E_{33}, E_{22}, E_{12}, E_{23}, E_{13}>.
\end{gathered}
$$

Example 3.3. $\mathfrak{g}=\mathfrak{g l}(3)$.

$$
e=E_{13}=\left(\begin{array}{ccc}
0 & 0 & 1 \\
0 & 0 & 0 \\
0 & 0 & 0
\end{array}\right), \quad h=\left(\begin{array}{ccc}
1 & 0 & 0 \\
0 & 1 & 0 \\
0 & 0 & -1
\end{array}\right) .
$$

$h$ defines a good but non-Dynkin $\mathbb{Z}$-grading for e, whose degrees on the elementary matrices $E_{i j}$ are

$$
\left(\begin{array}{ccc}
0 & 0 & 2 \\
0 & 0 & 2 \\
-2 & -2 & 0
\end{array}\right)
$$

\section{Definition of finite $W$-algebras}

Let $\mathfrak{g}$ be a reductive Lie algebra, $(\cdot \mid \cdot)$ be a non-degenerate invariant symmetric bilinear form, $e$ be a nilpotent element. Let $\mathfrak{g}=\oplus_{j \in \mathbb{Z}} \mathfrak{g}_{j}$ be a good $\mathbb{Z}$-grading for $e$. Let $\chi \in \mathfrak{g}^{*}$ by defined as follows: $\chi(x):=(x \mid e)$ for $x \in \mathfrak{g}$. 
Define a bilinear form on $\mathfrak{g}_{-1}$ by

$$
(x, y):=([x, y] \mid e)=\chi([x, y]) \text { for } x, y \in \mathfrak{g}_{-1} .
$$

Remark 4.1. The bilinear form on $\mathfrak{g}_{-1}$ is skew-symmetric and non-degenerate.

Proof. The skew-symmetry follows by definition.

The non-degeneracy follows from the bijection

$$
\operatorname{ad}(e): \mathfrak{g}_{-1} \longrightarrow \mathfrak{g}_{1}
$$

and the identity

$$
(x, y)=([x, y] \mid e)=(x \mid[y, e])
$$

Hence $\operatorname{dim} \mathfrak{g}_{-1}$ is even. Pick a Lagrangian (i.e. a maximal isotropic) subspace $\mathfrak{l}$ of $\mathfrak{g}_{-1}$ with respect to the form $(\cdot, \cdot)$. Then $\operatorname{dim} \mathfrak{l}=\frac{1}{2} \operatorname{dim} \mathfrak{g}_{-1}$.

Let $\mathfrak{m}=\left(\oplus_{j \leq-2} \mathfrak{g}_{j}\right) \oplus \mathfrak{l}$. Note that $\mathfrak{m}$ is a nilpotent subalgebra of $\mathfrak{g}$. The restriction of $\chi$ to $\mathfrak{m}$

$$
\chi: \mathfrak{m} \longrightarrow \mathbb{C}
$$

defines a one-dimensional representation $\mathbb{C}_{\chi}=<v>$ of $\mathfrak{m}$. In fact, if $x \in \mathfrak{g}_{i}, y \in \mathfrak{g}_{j}$ and $i \leq-2$ or $j \leq-2$, then $\chi([x, y])=0$ by Proposition 1 . If $x, y \in \mathfrak{l} \subset \mathfrak{g}_{-1}$, we have that $\chi([x, y])=(x, y)=0$ thanks to the Lagrangian condition on $\mathfrak{l}$.

Let $I_{\chi}$ be the left ideal of $U(\mathfrak{g})$ generated by $a-\chi(a)$ for all $a \in \mathfrak{m}$.

Definition 4.1. The generalized Whittaker module is

$$
Q_{\chi}:=U(\mathfrak{g}) \otimes_{U(\mathfrak{m})} \mathbb{C}_{\chi} \cong U(\mathfrak{g}) / I_{\chi}
$$

Definition 4.2. [Prl]. The finite $W$-algebra associated to the nilpotent element $e$ is

$$
W_{\chi}:=\operatorname{End}_{U(\mathfrak{g})}\left(Q_{\chi}\right)^{o p} .
$$

Example 4.1. Let $e=0$. Then $\chi=0, \mathfrak{g}_{0}=\mathfrak{g}, \mathfrak{m}=0$,

$$
Q_{\chi}=U(\mathfrak{g}), \quad W_{\chi}=U(\mathfrak{g}) .
$$

Theorem 4.1. B. Kostant (1978) [Ko].

For a regular nilpotent element $e \in \mathfrak{g}, W_{\chi} \cong Z(\mathfrak{g})$, the center of $U(\mathfrak{g})$.

Remark 4.2. The isoclasses of finite $W$-algebras do not depend on good $\mathbb{Z}$ grading ([BG]) and Lagrangian subspace $\mathfrak{l}([G G])$. 


\section{The Whittaker model definition of finite $W$-algebras}

We have given endomorphism algebra definition of $W_{\chi}$. Note that $W_{\chi}$ can also be identified as the space of Whittaker vectors in $U(\mathfrak{g}) / I_{\chi}$.

Definition 5.1. $A \mathfrak{g}$-module $L$ is called a Whittaker module if $a-\chi(a)$ for all $a \in \mathfrak{m}$ acts on L locally nilpotently. A Whittaker vector in a Whittaker $\mathfrak{g}$-module $L$ is a vector $x \in L$ which satisfies $(a-\chi(a)) x=0$ for all $a \in \mathfrak{m}$.

Proposition 5.1. (see $[W])$. Let $\pi: U(\mathfrak{g}) \rightarrow U(\mathfrak{g}) / I_{\chi}$ be the natural projection, and let $y \in U(\mathfrak{g})$. Then

$$
W_{\chi}=\left\{\pi(y) \in U(\mathfrak{g}) / I_{\chi} \mid(a-\chi(a)) y \in I_{\chi} \text { for all } a \in \mathfrak{m}\right\} .
$$

Proof. Since $Q_{\chi}=U(\mathfrak{g}) / I_{\chi}$ is a cyclic module, then any endomorphism of the $\mathfrak{g}$-module $Q_{\chi}$ is determined by the image of $v$. The image of $v$ must be annihilated by $I_{\chi}$. Hence $W_{\chi}$ can be identified as the space of Whittaker vectors in $U(\mathfrak{g}) / I_{\chi}$.

By definition of $I_{\chi}, W_{\chi}$ can be further identified with the subspace of $a d \mathfrak{m}$ invariants in $Q_{\chi}$ :

$$
W_{\chi}=\left(Q_{\chi}\right)^{a d \mathfrak{m}}:=\left\{\pi(y) \in U(\mathfrak{g}) / I_{\chi} \mid[a, y] \in I_{\chi} \text { for all } a \in \mathfrak{m}\right\}
$$

The algebra structure on $W_{\chi}$ is given by

$$
\pi\left(y_{1}\right) \pi\left(y_{2}\right)=\pi\left(y_{1} y_{2}\right)
$$

for $y_{i} \in U(\mathfrak{g})$ such that $\left[a, y_{i}\right] \in I_{\chi}$ for all $a \in \mathfrak{m}$ and $i=1,2$.

Exercise 2. Check directly that

(1) The ideal $I_{\chi}$ is ad $\mathfrak{m}$-invariant, hence $\left(Q_{\chi}\right)^{a d \mathfrak{m}}$ as a vector space is welldefined.

(2) For $y_{i}$ satisfying $\left[a, y_{i}\right] \in I_{\chi}$ for all $a \in \mathfrak{m}$ and $i=1$, 2, we have $\left[a, y_{1} y_{2}\right] \in I_{\chi}$ for all $a \in \mathfrak{m}$. Hence $\left(Q_{\chi}\right)^{a d \mathfrak{m}}$ as an algebra is well-defined.

\section{Finite $W$-algebras for even good $\mathbb{Z}$-gradings}

Definition 6.1. A good $\mathbb{Z}$-grading $\mathfrak{g}=\oplus_{j \in \mathfrak{g}} \mathfrak{g}_{j}$ is called even, if $\mathfrak{g}_{j}=0$ unless $j$ is an even integer.

The definition of $W_{\chi}$ for an even good $\mathbb{Z}$-grading is simpler, since in this case $\mathfrak{g}_{-1}=0$. Hence there is no complications of choice of a Lagrangian subspace $\mathfrak{l}$ and $\mathfrak{m}=\oplus_{j \leq-2} \mathfrak{g}_{j}$. 
Let $\mathfrak{p}:=\oplus_{j \geq 0} \mathfrak{g}_{j}$ be a parabolic subalgebra of $\mathfrak{g}$. From the PBW theorem,

$$
U(\mathfrak{g})=U(\mathfrak{p}) \oplus I_{\chi} .
$$

The projection

$$
p r_{\chi}: U(\mathfrak{g}) \longrightarrow U(\mathfrak{p})
$$

along this direct sum decomposition induces an isomorphism:

$$
\overline{p r}_{\chi}: U(\mathfrak{g}) / I_{\chi} \stackrel{\sim}{\longrightarrow} U(\mathfrak{p}) .
$$

The algebra $W_{\chi}$ can be regarded as a subalgebra of $U(\mathfrak{p})$.

Consider a $\chi$-twisted adjoint action of $\mathfrak{m}$ on $U(\mathfrak{p})$ by

$$
a \cdot y:=p r_{\chi}([a, y]) \text { for } a \in \mathfrak{m} \text { and } y \in U(\mathfrak{p}) .
$$

Identify $W_{\chi}$ as

$$
W_{\chi}=U(\mathfrak{p})^{a d \mathfrak{m}}:=\left\{y \in U(\mathfrak{p}) \mid[a, y] \in I_{\chi} \text { for all } a \in \mathfrak{m}\right\} .
$$

Since $\overline{p r}_{\chi}: U(\mathfrak{g}) / I_{\chi} \stackrel{\sim}{\longrightarrow} U(\mathfrak{p})$ is an isomorphism of $\mathfrak{m}$-modules, this definition is equivalent to the definition (1) (for even $\mathbb{Z}$-gradings) [W].

\section{Filtration on $W_{\chi}$}

To introduce a filtration on $W_{\chi}$, first we will recall the definition of a filtered algebra [A].

Definition 7.1. A filtered algebra is an algebra A, which has an increasing sequence of subspaces such that

$$
\{0\} \subset F_{0} \subset F_{1} \subset \ldots \subset F_{i} \subset \ldots A, \quad A=\bigcup_{i} F_{i},
$$

which is compatible with the multiplication:

$$
F_{i} \cdot F_{j} \subset F_{i+j} \text { for all } i, j \text {. }
$$

As a vector space the associated graded algebra is

$$
\operatorname{Gr}(A):=\bigoplus_{i} G_{i}
$$

where

$$
G_{i}=F_{i} / F_{i-1} \text { for alli }>0, \quad G_{0}=F_{0},
$$

with multiplication

$$
\left(x+F_{i-1}\right)\left(y+F_{j-1}\right)=x y+F_{i+j-1}, \quad x \in F_{i}, \quad y \in F_{j} .
$$


Definition 7.2. Kazhdan filtration on $W_{\chi}$.

Let $\mathfrak{g}$ be a reductive Lie algebra with a Dynkin $\mathbb{Z}$-grading: $\mathfrak{g}=\oplus_{j \in \mathbb{Z}} \mathfrak{g}_{j}$, and let $\mathfrak{m}=\left(\oplus_{j \leq-2} \mathfrak{g}_{j}\right) \oplus \mathfrak{l}$. Let $\mathfrak{n} \subset \mathfrak{g}$ be an ad $(h)$-invariant subspace such that $\mathfrak{g}=\mathfrak{m} \oplus \mathfrak{n}$ and $\mathbb{C}_{\chi}=<v>$ be one-dimensional representation of $\mathfrak{m}$. Then

$$
W_{\chi}=\{X \in U(\mathfrak{g}) / U(\mathfrak{g}) \mathfrak{m} \cong S(\mathfrak{n}) \mid a X v=\chi(a) X v \text { for all } a \in \mathfrak{m}\} .
$$

For any $y \in \mathfrak{n}$, let $w t(y)$ be the weight of $y$ with respect to $a d(h)$ and

$$
\operatorname{deg}(y)=w t(y)+2
$$

The degree function deg induces a $\mathbb{Z}$-grading on $S(\mathfrak{n})$. This grading defines a filtration on $W_{\chi}$.

Theorem 7.1. A. Premet [Prl].

The associated graded algebra $G r\left(W_{\chi}\right)$ is isomorphic to $S\left(\mathfrak{g}^{e}\right)$.

Idea of Proof. Introduce the map

$$
P: W_{\chi} \longrightarrow S\left(\mathfrak{g}^{e}\right) .
$$

For $X \in W_{\chi} \subset S(\mathfrak{n})$, let $P(X)$ be the highest weight component in the highest degree component of $X$, then $P(X)$ belongs to $S\left(\mathfrak{g}^{e}\right)$.

Example 7.1. If $e=0$, then the statement of Theorem 3 is the PBW theorem. In this case $W_{\chi}=U(\mathfrak{g}), \mathfrak{g}^{e}=\mathfrak{g}$ and we have that

$$
\operatorname{Gr}(U(\mathfrak{g})) \cong S(\mathfrak{g}) .
$$

The finite $W$-algebras associated to regular nilpotent elements are called the principal finite $W$-algebras.

\section{The case of $\mathfrak{g}=\mathfrak{g l}(2)$}

In this section, we describe the principal finite $W$-algebra for $\mathfrak{g l}(2)$.

Form: $(a \mid b)=\operatorname{tr}(a b)$.

$$
e=\left(\begin{array}{ll}
0 & 1 \\
0 & 0
\end{array}\right), \quad f=\left(\begin{array}{ll}
0 & 0 \\
1 & 0
\end{array}\right), \quad h=\left(\begin{array}{cc}
1 & 0 \\
0 & -1
\end{array}\right) .
$$

Note that $e$ is a regular nilpotent element, $h$ defines an even Dynkin $\mathbb{Z}$-grading of $\mathfrak{g}$ whose degrees on the elementary matrices $E_{i j}$ are

$$
\left(\begin{array}{cc}
0 & 2 \\
-2 & 0
\end{array}\right)
$$

Let $z=\left(\begin{array}{ll}1 & 0 \\ 0 & 1\end{array}\right)$, then $z$ is a central element of $\mathfrak{g}$.

$$
\mathfrak{g}^{e}=<z, e>, \quad \operatorname{dim} \mathfrak{g}^{e}=\operatorname{dim} \mathfrak{g}_{0}=2 .
$$




$$
\begin{gathered}
\mathfrak{m}=\mathfrak{g}_{-2}=<f>, \quad \chi(f)=(f \mid e)=1, \\
\mathfrak{n}=\mathfrak{p}=\mathfrak{g}_{0} \oplus \mathfrak{g}_{2}, \quad \mathbb{C}_{\chi}=<v>.
\end{gathered}
$$

$W_{\chi}$ is the polynomial algebra generated by $\pi(z)$ and $\pi\left(e+\frac{1}{4} h^{2}-\frac{1}{2} h\right)$.

$$
\Omega=e f+f e+\frac{1}{2} h^{2} \in Z(\mathfrak{g}) \text { is the quadratic Cazimir element of } \mathfrak{g} .
$$

We have that

$$
\left.\left.\frac{1}{2} \pi(\Omega)=\frac{1}{2} \pi\left(e f+f e+\frac{1}{2} h^{2}\right)\right)=\frac{1}{2} \pi\left(2 e f-h+\frac{1}{2} h^{2}\right)\right)=\pi\left(e+\frac{1}{4} h^{2}-\frac{1}{2} h\right) .
$$

The generators of $W_{\chi}$ can be identified with elements of $\mathfrak{g}^{e}$ :

$$
\begin{gathered}
\pi(z) \stackrel{P}{\longrightarrow} z, \\
\frac{1}{2} \pi(\Omega) \stackrel{P}{\longrightarrow} e .
\end{gathered}
$$

\section{The case of $\mathfrak{g}=\mathfrak{g l}(n)$}

In this section, we describe the principal finite $W$-algebra for $\mathfrak{g l}(n)$.

Form: $(a \mid b)=\operatorname{tr}(a b)$. Let

$$
e=\left(\begin{array}{cccccc}
0 & 1 & 0 & 0 & 0 & 0 \\
0 & 0 & 1 & 0 & 0 & 0 \\
0 & 0 & 0 & \cdots & 0 & 0 \\
0 & 0 & 0 & 0 & 1 & 0 \\
0 & 0 & 0 & 0 & 0 & 1 \\
0 & 0 & 0 & 0 & 0 & 0
\end{array}\right), \quad f=\left(\begin{array}{cccccc}
0 & 0 & 0 & 0 & 0 & 0 \\
n-1 & 0 & 0 & 0 & 0 & 0 \\
0 & 2(n-2) & 0 & \cdots & 0 & 0 \\
0 & 0 & 0 & 0 & 0 & 0 \\
0 & 0 & 0 & 0 & 0 & 0 \\
0 & 0 & 0 & 0 & n-1 & 0
\end{array}\right),
$$

$h=\operatorname{diag}(n-1, n-3, \ldots, 3-n, 1-n)$. Note that $e$ is a regular nilpotent element, and $h$ defines an even Dynkin $\mathbb{Z}$-grading of $\mathfrak{g}$ whose degrees on the elementary matrices $E_{i j}$ are

$$
\left(\begin{array}{cccccc}
0 & 2 & 4 & 6 & \cdots & 2 n-2 \\
-2 & 0 & 2 & 4 & \cdots & 2 n-4 \\
-4 & -2 & 0 & 2 & \cdots & 2 n-6 \\
-6 & -4 & -2 & 0 & \cdots & 2 n-8 \\
\cdots & \cdots & \cdots & \cdots & \cdots & \cdots \\
2-2 n & \cdots & -6 & -4 & -2 & 0
\end{array}\right)
$$

Let $z=\operatorname{diag}(1, \ldots, 1)$ be a central element of $\mathfrak{g l}(n)$. Then

$$
\begin{gathered}
\mathfrak{g}^{e}=<z, e, e^{2}, e^{3}, \ldots, e^{n-1}>, \quad \operatorname{dim} \mathfrak{g}^{e}=\operatorname{dim} \mathfrak{g}_{0}=n . \\
\mathfrak{m}=\bigoplus_{j \geq 2}^{n} \mathfrak{g}_{2-2 j}, \quad \chi\left(E_{i+1, i}\right)=1, \quad \chi\left(E_{i+k, i}\right)=0 \text { if } k \geq 2 .
\end{gathered}
$$


Recall that the Casimir elements of $\mathfrak{g}$ are generators of $Z(\mathfrak{g})$, the center of $U(\mathfrak{g})$. If $\mathfrak{g}=\mathfrak{g l}(n)$ they are given as follows:

$$
\Omega_{k}=\sum_{i_{1}, i_{2}, \ldots, i_{k}} E_{i_{1} i_{2}} E_{i_{2} i_{3}} \ldots E_{i_{k} i_{1}}, \text { for } k \geq 1
$$

Note that $W_{\chi}$ is the polynomial algebra generated by $n$ elements:

$$
\pi(z), \pi\left(\Omega_{2}\right), \pi\left(\Omega_{3}\right), \ldots, \pi\left(\Omega_{n}\right)
$$

The generators of $W_{\chi}$ can be identified with elements of $\mathfrak{g}^{e}$ :

$$
\begin{aligned}
& \pi(z) \stackrel{P}{\longrightarrow} z, \\
& \frac{1}{k} \pi\left(\Omega_{k}\right) \stackrel{P}{\longrightarrow} e^{k-1} \text { for } k=2, \ldots, n .
\end{aligned}
$$

\section{Lie superalgebras}

Let $\mathbb{Z}_{2}=\mathbb{Z} / 2 \mathbb{Z}=\{\overline{0}, \overline{1}\}$.

Definition 10.1. A superspace is a $\mathbb{Z}_{2}$-graded vector space

\section{Definition 10.2.}

$$
V=V_{\overline{0}} \oplus V_{\overline{1}} .
$$

The dimension of a superspace: $\operatorname{dim} V=(m \mid n)$, where $\operatorname{dim} V_{\overline{0}}=m, \operatorname{dim} V_{\overline{0}}=n$.

The parity of an element: if $v \in V_{\overline{0}}$ then $p(v)=\overline{0}$, if $v \in V_{\overline{1}}$ then $p(v)=\overline{1}$.

Definition 10.3. A superalgebra is a $\mathbb{Z}_{2}$-graded algebra

$$
A=A_{\overline{0}} \oplus A_{\overline{1}}, \quad A_{i} A_{j} \subseteq A_{i+j} \text { for } i, j \in \mathbb{Z}_{2} .
$$

Definition 10.4. A Lie superalgebra is a $\mathbb{Z}_{2}$-graded algebra

$$
\mathfrak{g}=\mathfrak{g}_{\overline{0}} \oplus \mathfrak{g}_{\overline{1}}
$$

with an operation [, ] satisfying the following axioms:

1. super-anticommutativity:

$$
[x, y]=-(-1)^{p(x) p(y)}[y, x] \quad \text { for all } x, y \in \mathfrak{g},
$$

2. super-Jacobi identity:

$$
[x,[y, z]]=[[x, y], z]+(-1)^{p(x) p(y)}[y,[x, z]] \quad \text { for all } x, y, z \in \mathfrak{g} .
$$

Remark 10.1. $\mathfrak{g}_{\overline{0}}$ is a Lie algebra, $\left[\mathfrak{g}_{\overline{0}}, \mathfrak{g}_{\overline{1}}\right] \subseteq \mathfrak{g}_{\overline{1}}$, hence $\mathfrak{g}_{\overline{1}}$ is a module over $\mathfrak{g}_{\overline{0}}$, $\left[\mathfrak{g}_{\overline{1}}, \mathfrak{g}_{\overline{1}}\right] \subseteq \mathfrak{g}_{\overline{0}}$.

Definition 10.5. A Lie superalgebra $\mathfrak{g}$ is simple, if it is not abelian and the only $\mathbb{Z}_{2}$-graded ideals of $\mathfrak{g}$ are $\{0\}$ and $\mathfrak{g}$. 
Remark 10.2. Sign Rule. If an element $x$ with parity $p(x)$ moves through an element $y$ with parity $p(y)$, then the sign $(-1)^{p(x) p(y)}$ appears in the formula.

\section{Main examples of Lie superalgebras}

Example 11.1. The General Linear Lie superalgebra.

$$
\begin{gathered}
\mathfrak{g}=\mathfrak{g l}(m \mid n)=\mathfrak{g}_{\overline{0}} \oplus \mathfrak{g}_{\overline{1}}, \text { where } \\
\mathfrak{g}_{\overline{0}}=\left\{\left(\begin{array}{c|c}
A & 0 \\
\hline 0 & B
\end{array}\right) \mid \text { A is a } m \times \text { m matrix, B is a } n \times n \text { matrix }\right\}, \\
\mathfrak{g}_{\overline{1}}=\left\{\left(\begin{array}{c|c}
0 & C \\
\hline D & 0
\end{array}\right) \mid \text { C is a } m \times n \text { matrix, } D \text { is a } n \times m \text { matrix }\right\} .
\end{gathered}
$$

Definition 11.1. Super-bracket:

$$
[X, Y]=X Y-(-1)^{p(X) p(Y)} Y X \text { for } X, Y \in \mathfrak{g} .
$$

Super-trace:

$$
\operatorname{str}\left(\begin{array}{l|l}
A & C \\
\hline D & B
\end{array}\right)=\operatorname{tr}(A)-\operatorname{tr}(B) .
$$

Example 11.2. The Special Linear Lie superalgebra:

$$
\mathfrak{s l}(m \mid n):=\{X \in \mathfrak{g l}(m \mid n) \mid \operatorname{str} X=0\} .
$$

Note that $\mathfrak{s l}(m \mid n)$ is simple if and only if $m \neq n$. If $m=n$ then $\mathfrak{s l}(n \mid n) /<1_{2 n}>$ is simple.

Example 11.3. The Orthogonal-Symplectic Lie superalgebra.

Let $F$ be a non-degenerate super symmetric bilinear form on a superspace $V=V_{\overline{0}} \oplus V_{\overline{1}}$, where $\operatorname{dim} V=(m \mid n)$ :

$$
F(v, w)=(-1)^{p(v) p(w)} F(w, v) \quad v, w \in V .
$$

The restriction of $F$ to $V_{\overline{0}}$ is symmetric, and to $V_{\overline{1}}$ is skew-symmetric, hence $n$ is even.

$\mathfrak{v} \mathfrak{s p}(m \mid n):=\left\{X \in \mathfrak{g l}(m \mid n) \mid F(X(v), w)+(-1)^{p(X) p(v)} F(v, X(w))=0, \forall v, w \in V\right\}$.

For instance, let $m=2 l, \quad n=2 r$.

$$
F=\left(\begin{array}{cc|cc}
0 & 1_{l} & 0 & 0 \\
1_{l} & 0 & 0 & 0 \\
\hline 0 & 0 & 0 & 1_{r} \\
0 & 0 & -1_{r} & 0
\end{array}\right),
$$

São Paulo J.Math.Sci. 7, 1 (2013), 1-32 
where $1_{l}$ is the identity $l \times l$-matrix, $1_{r}$ is the identity $r \times r$-matrix. Then

$$
\mathfrak{v} \mathfrak{s p}(m \mid n)=\left(\begin{array}{cc|cc}
A & B & X & P \\
C & -A^{t} & Y & Q \\
\hline Q^{t} & P^{t} & D & E \\
-Y^{t} & -X^{t} & F & -D^{t}
\end{array}\right),
$$

where $A$ is any $l \times l$ matrix, $B$ and $C$ are skew-symmetric $l \times l$ matrices, $D$ is any $r \times r$ matrix, $E$ and $F$ are symmetric $r \times r$ matrices, $X, Y, P, Q$ are any $l \times r$ matrices.

\section{Classification of simple finite-dimensional Lie superalgebras}

Definition 12.1. [K]. A Lie superalgebra $\mathfrak{g}=\mathfrak{g}_{\overline{0}} \oplus \mathfrak{g}_{\overline{1}}$ is called classical, if it is simple, and the representation of the Lie algebra $\mathfrak{g}_{0}$ on $\mathfrak{g}_{1}$ is completely reducible.

Definition 12.2. A classical Lie superalgebra $\mathfrak{g}$ is called basic, if $\mathfrak{g}$ admits an even non-degenerate $\mathfrak{g}$-invariant bilinear form.

Definition 12.3. A classical Lie superalgebra $\mathfrak{g}$ is of Type $I$, if $\mathfrak{g}_{\overline{1}}$ is a direct sum if two simple $\mathfrak{g}_{0}$-submodules.

Remark 12.1. Notations:

$$
\begin{aligned}
& A(m, n)=\mathfrak{s l}(m+1 \mid n+1) \text { for } m \neq n, \quad m, n \geq 0, \\
& A(n, n)=\mathfrak{s l}(n+1 \mid n+1) /<1_{2 n+2}>, \quad n>0, \\
& B(m, n)=\mathfrak{v} \mathfrak{s p}(2 m+1 \mid 2 n), \quad m \geq 0, n>0, \\
& D(m, n)=\mathfrak{v} \mathfrak{s}(2 m \mid 2 n), \quad m \geq 2, n>0, \\
& C(n)=\mathfrak{v} \mathfrak{s p}(2 \mid 2 n-2), \quad n \geq 2 .
\end{aligned}
$$

Theorem 12.1. V. G. Kac (1977) [K].

A simple finite-dimensional Lie superalgebra over an algebraically closed field of characteristic zero is isomorphic either to one of the simple Lie algebras, or to one of classical Lie superalgebras:

$$
A(m, n), B(m, n), C(n), D(m, n), D(2,1 ; \alpha), F(4), G(3), P(n), \tilde{Q}(n),
$$

or to one of Lie superalgebras of Cartan type: $W(n), S(n), H(n), \tilde{S}(n)$.

\section{Finite $W$-algebras for Lie superalgebras (joint work with V. Serganova)}

Finite $W$-algebras for Lie superalgebras have been studied by C. Briot, E. Ragoucy, J. Brundan, J. Brown, S. Goodwin, W. Wang, L. Zhao and other mathematicians and physicists [BR, BBG, W, Z]. Analogues of finite $W$-algebras for Lie superalgebras in terms of BRST cohomology were defined in [DK]. 
We consider the case when $\mathfrak{g}$ is a classical simple Lie superalgebra, i.e. $\mathfrak{g}=$ $\mathfrak{g}_{\overline{0}} \oplus \mathfrak{g}_{\overline{1}}, \mathfrak{g}_{\overline{0}}$ is a reductive Lie algebra, and $\mathfrak{g}$ has an invariant super-symmetric bilinear form $(\cdot \mid \cdot)$.

Remark 13.1. The Premet's definition makes sense for Lie superalgebras. One should consider a good $\mathbb{Z}$-grading $\mathfrak{g}=\oplus_{j \in \mathbb{Z}} \mathfrak{g}_{j}$ for $e \in\left(\mathfrak{g}_{2}\right)_{\overline{0}}$ (i.e. a $\mathbb{Z}$-grading which satisfies properties (2) and (3) of Def. 6). Note that good $\mathbb{Z}$-gradings of basic Lie superalgebras over an algebraically closed field of characteristic zero were classified in $[H]$.

Let $e \in \mathfrak{g}_{\overline{0}}$ be an even nilpotent element, and we fix $\mathfrak{s l}(2)=<e, h, f>$. As in the Lie algebra case, the linear operator $a d(h)$ defines a $\mathbb{Z}$-grading $\mathfrak{g}=\oplus_{j \in \mathbb{Z}} \mathfrak{g}_{i}$. Let $\mathfrak{g}^{e}:=\operatorname{Ker}(\operatorname{ad}(e))$. Note that $\operatorname{dim} \mathfrak{g}^{e}=\operatorname{dim} \mathfrak{g}_{0}+\operatorname{dim} \mathfrak{g}_{1}$. Let $\mathfrak{l}$ be a Lagrangian subspace in $\mathfrak{g}_{-1}$, with respect to the super-skew-symmetric bilinear form $(x, y)=$ $([x, y] \mid e)$. We consider a nilpotent subalgebra $\mathfrak{m}=\oplus_{j \leq-2} \mathfrak{g}_{j} \oplus \mathfrak{l}$ of $\mathfrak{g}$. Let $\chi \in \mathfrak{g}^{*}$ be defined by $\chi(x)=(x \mid e)$. Let $\mathbb{C}_{\chi}=\langle v\rangle$ be the one-dimensional m-module with character $\chi$. Let $I_{\chi}$ be the left ideal of $U(\mathfrak{g})$ generated by $a-\chi(a)$ for all $a \in \mathfrak{m}$.

The generalized Whittaker module $Q_{\chi}$ is defined as in Def.8. The finite $W$ algebra $W_{\chi}$ associated to the nilpotent element $e$ is defined as in Def.9.

\section{Remark 13.2.}

(1) The isoclasses of $W_{\chi}$ do not depend on the choice of Lagrangian subspace $\mathfrak{l}$ [Z].

(2) Theorem of Kostant does not hold for Lie superalgebras, since $W_{\chi}$ must have a non-trivial odd part, and the center $Z(\mathfrak{g})$ of $U(\mathfrak{g})$ is even.

(3) Kazhdan filtration on $W_{\chi}$ can be defined exactly as in the Lie algebra case (see Def. 13).

Proposition 13.1. $G r\left(W_{\chi}\right)$ is supercommutative.

Remark 13.3. If $\operatorname{dim}\left(\mathfrak{g}_{-1}\right)_{\overline{1}}$ is even, then one can construct the similar map

$$
P: W_{\chi} \longrightarrow S\left(\mathfrak{g}^{e}\right)
$$

by taking the highest weight component in the highest degree component.

If $\operatorname{dim}\left(\mathfrak{g}_{-1}\right)_{\overline{1}}$ is odd, then there exists an odd element $\theta$ in $\mathfrak{g}_{-1} \cap \mathfrak{l}^{\perp}$ such that $\pi(\theta) \in W_{\chi}$ and $\pi(\theta)^{2}=1$.

In what follows we study the principal finite $W$-algebras, which are the finite $W$-algebras associated to even regular nilpotent elements.

\section{Defect}

Definition 14.1. $[K W]$. 
Let $\mathfrak{g}$ be a classical Lie superalgebra, and let $\triangle$ be the set of roots with respect to a maximal torus in $\mathfrak{g}_{\overline{0}}$. If $\mathfrak{g}$ is a basic Lie superalgebra, then the defect of $\mathfrak{g} \operatorname{def}(\mathfrak{g})$ is the dimension of a maximal isotropic subspace in the $\mathbb{R}$-span of $\triangle$.

\section{Example 14.1.}

$$
\begin{gathered}
\operatorname{def}(\mathfrak{s l}(m \mid n))=\min (m, n), \\
\operatorname{def}(\mathfrak{v} \mathfrak{s p}(2 m \mid 2 n))=\operatorname{def}(\mathfrak{v} \mathfrak{s p}(2 m+1 \mid 2 n))=\min (m, n) .
\end{gathered}
$$

The exceptional Lie superalgebras

$$
D(2,1 ; \alpha), G(3), F(4)
$$

have defect one.

Remark 14.1. [PS1]. If e is a regular nilpotent element in $\mathfrak{g}$, then

$$
\operatorname{dim}\left(\mathfrak{g}^{e}\right)_{\overline{1}}=2 \operatorname{def}(\mathfrak{g}) \text { or } 2 \operatorname{def}(\mathfrak{g})+1 .
$$

$\operatorname{dim}\left(\mathfrak{g}^{e}\right)_{\overline{1}}=2 \operatorname{def}(\mathfrak{g})$, if $\mathfrak{g}=\mathfrak{s l}(m \mid n), \mathfrak{v} \mathfrak{s p}(2 m+1 \mid 2 n), m \geq n ; \mathfrak{v} \mathfrak{s p}(2 m \mid 2 n)$, $m \leq n, G(3)$;

$\operatorname{dim}\left(\mathfrak{g}^{e}\right)_{\overline{1}}=2 \operatorname{def}(\mathfrak{g})+1$, if $\mathfrak{g}=\mathfrak{v} \mathfrak{s p}(2 m+1 \mid 2 n), m<n ; \mathfrak{v} \mathfrak{p} \mathfrak{p}(2 m \mid 2 n), m>n ;$ $D(2,1 ; \alpha), F(4)$.

\section{The case of $\mathfrak{g}=\mathfrak{s l}(1 \mid n)$}

In this section, we describe the principal finite $W$-algebra for $\mathfrak{s t}(1 \mid n)$ and more generally, for a Lie superalgebra of Type I and defect one.

Form: $(a \mid b)=-\operatorname{str}(a b)$

$$
\begin{gathered}
e=\left(\begin{array}{c|ccccc}
0 & 0 & 0 & 0 & 0 & 0 \\
\hline 0 & 0 & 1 & 0 & 0 & 0 \\
0 & 0 & 0 & \cdots & 0 & 0 \\
0 & 0 & 0 & 0 & 1 & 0 \\
0 & 0 & 0 & 0 & 0 & 1 \\
0 & 0 & 0 & 0 & 0 & 0
\end{array}\right), f=\left(\begin{array}{c|c|ccccc}
0 & 0 & 0 & 0 & 0 & 0 \\
\hline 0 & 0 & 0 & 0 & 0 & 0 \\
0 & n-1 & 0 & \cdots & 0 & 0 \\
0 & 0 & 2(n-2) & 0 & 0 & 0 \\
0 & 0 & 0 & 0 & 0 & 0 \\
0 & 0 & 0 & 0 & n-1 & 0
\end{array}\right) \\
h=\operatorname{diag}(0 \mid n-1, n-3, \ldots, 3-n, 1-n) .
\end{gathered}
$$

Note that $e$ is a regular nilpotent element. We will use the following notations for some elementary matrices in $\mathfrak{g l}(1 \mid n)$ :

$$
E_{i j}=\left(\begin{array}{c|ccccc}
h_{0} & \mu_{1} & \mu_{2} & \mu_{3} & \cdots & \mu_{n} \\
\hline \xi_{1} & h_{1} & e_{1} & \cdots & \cdots & \cdots \\
\xi_{2} & f_{1} & h_{2} & e_{2} & \cdots & \cdots \\
\xi_{3} & \cdots & f_{2} & h_{3} & \cdots & \cdots \\
\cdots & \cdots & \cdots & \cdots & \cdots & e_{n-1} \\
\xi_{n} & \cdots & \cdots & \cdots & f_{n-1} & h_{n}
\end{array}\right) .
$$


$h$ defines a Dynkin $\mathbb{Z}$-grading of $\mathfrak{g}$ whose degrees on the elementary matrices $E_{i j}$ are

$$
\left(\begin{array}{c|ccccc}
0 & 1-n & 3-n & \cdots & n-3 & \mathbf{n}-\mathbf{1} \\
\hline \mathbf{n}-\mathbf{1} & 0 & 2 & 4 & \cdots & 2 n-2 \\
n-3 & -2 & 0 & 4 & \cdots & 2 n-4 \\
\cdots & \cdots & \cdots & \cdots & \cdots & \cdots \\
3-n & 4-2 n & \cdots & \cdots & 0 & 2 \\
1-n & 2-2 n \cdots & \cdots & \cdots & -2 & 0
\end{array}\right) .
$$

Let $c=\operatorname{diag}(n \mid 1, \cdots, 1)$ be a central elements of $\mathfrak{g}_{0}$. Then

$$
\mathfrak{g}^{e}=<e, e^{2}, \cdots, e^{n-1}, c \mid \xi_{1}, \mu_{n}>, \quad \operatorname{dim}\left(\mathfrak{g}^{e}\right)=(n \mid 2) .
$$

Let $\mathfrak{m}=\left(\bigoplus_{j \leq-2} \mathfrak{g}_{j}\right) \bigoplus \mathfrak{l}$. Note that if $n$ is odd, then $\mathfrak{l}=0$, and if $n=2 k$, then $\mathfrak{l}=<\xi_{k+1}>$. Note also that $\mathfrak{m}$ is generated by $f_{1}, \ldots, f_{n} ; \mu_{1}, \ldots, \mu_{k-1}, \xi_{k+1}, \ldots, \xi_{n}$, if $n=2 k$, and by $f_{1}, \ldots, f_{n} ; \mu_{1}, \ldots, \mu_{k}, \xi_{k+2}, \ldots, \xi_{n}$, if $n=2 k+1$.

$$
\chi\left(f_{i}\right)=1 \quad \chi\left(\mu_{i}\right)=\chi\left(\xi_{i}\right)=0 .
$$

Let $\mathfrak{n}$ be an $\operatorname{ad}(h)$-invariant subspace of $\mathfrak{g}: \mathfrak{g}=\mathfrak{m} \oplus \mathfrak{n}$. Recall that $\mathfrak{g}$ admits $\mathbb{Z}$ grading $\mathfrak{g}=\mathfrak{g}^{-1} \oplus \mathfrak{g}^{0} \oplus \mathfrak{g}^{1}$ consistent with the $\mathbb{Z}_{2}$-grading:

$$
\begin{gathered}
\mathfrak{g}^{0}=\left(\begin{array}{c|ccc}
* & 0 & \ldots & 0 \\
\hline 0 & * & * & * \\
\ldots & \ldots & \ldots & \ldots \\
0 & * & * & *
\end{array}\right), \quad \mathfrak{g}^{1}=\left(\begin{array}{cc|ccc}
0 & * & \ldots & * \\
\hline 0 & 0 & 0 & 0 \\
\ldots & \ldots & \ldots & \ldots \\
0 & 0 & 0 & 0
\end{array}\right) \\
\mathfrak{g}^{-1}=\left(\begin{array}{c|ccc}
0 & 0 & \ldots & 0 \\
\hline * & 0 & 0 & 0 \\
\ldots & \ldots & \ldots & \ldots \\
* & 0 & 0 & 0
\end{array}\right)
\end{gathered}
$$

Fix an $a d(h)$-homogeneous bases:

$B\left(\mathfrak{m}_{-1}\right)$ of $\mathfrak{m} \cap \mathfrak{g}^{-1}, B\left(\mathfrak{m}_{1}\right)$ of $\mathfrak{m} \cap \mathfrak{g}^{1}, B\left(\mathfrak{n}_{-1}\right)$ of $\mathfrak{n} \cap \mathfrak{g}^{-1}, B\left(\mathfrak{n}_{1}\right)$ of $\mathfrak{n} \cap \mathfrak{g}^{1}$.

Set

$$
\begin{aligned}
& R_{1}=\left(\prod_{x \in B\left(\mathfrak{m}_{1}\right)} x\right)\left(\prod_{y \in B\left(\mathfrak{n}_{-1}\right)} y\right), \\
& R_{2}=\left(\prod_{y \in B\left(\mathfrak{m}_{-1}\right)} y\right)\left(\prod_{x \in B\left(\mathfrak{n}_{1}\right)} x\right) .
\end{aligned}
$$

Note that $\pi\left(R_{1}\right)$ and $\pi\left(R_{2}\right)$ are both Whittaker vectors, hence $\pi\left(R_{1}\right), \pi\left(R_{2}\right) \in W_{\chi}$. Let

where $E_{i j}$ is an elementary matrix, and

$$
p\left(E_{i j}\right)=p(i)+p(j),
$$

$$
p(i)= \begin{cases}0 \quad \text { if } \quad 1 \leq i \leq m \\ 1 \quad \text { if } \quad m+1 \leq i \leq m+n\end{cases}
$$


Remark 15.1. Casimir elements of $\mathfrak{g l}(m \mid n)$ are

$$
\Omega_{k}=\sum_{i_{1}, i_{2}, \ldots, i_{k}}(-1)^{p\left(i_{2}\right)+\cdots+p\left(i_{k}\right)} E_{i_{1} i_{2}} E_{i_{2} i_{3}} \ldots E_{i_{k} i_{1}} .
$$

Even generators of $W_{\chi}$ are $\pi\left(\Omega_{k}\right)$, for $k=2, \ldots n$ and $\pi(c)$.

The generators of $W_{\chi}$ can be identified with elements of $\mathfrak{g}^{e}$ :

$$
\begin{aligned}
& (-1)^{k+1} \frac{1}{k} \pi\left(\Omega_{k}\right) \stackrel{P}{\longrightarrow} e^{k-1}, \quad k=2, \ldots, n, \\
& \pi(c) \stackrel{P}{\longrightarrow} c, \\
& \pi\left(R_{1}\right) \stackrel{P}{\longrightarrow} \xi_{1}, \\
& \pi\left(R_{2}\right) \stackrel{P}{\longrightarrow} \mu_{n} .
\end{aligned}
$$

Theorem 15.1. [PS1]. Let $\mathfrak{g}$ be a Lie superalgebra of Type I and defect one

(i.e. $\mathfrak{g}=\mathfrak{s l}(1 \mid n)$ or $\mathfrak{v} \mathfrak{s p}(2 \mid 2 n-2)$ ). Let $n$ be the rank of $\mathfrak{g}_{\hat{0}}, c$ be a central element of $\mathfrak{g}_{0}$, and $\Omega_{2}, \ldots, \Omega_{n}$ be the first $n-1$ Casimir elements in $Z(\mathfrak{g})$. Then the principal finite $W$-algebra $W_{\chi}$ is a finite extension of $\mathbb{C}\left[\pi(c), \pi\left(\Omega_{2}\right), \ldots, \pi\left(\Omega_{n}\right)\right]$ with odd generators $\pi\left(R_{1}\right), \pi\left(R_{2}\right)$ and defining relations

$$
\begin{gathered}
\pi\left(R_{1}\right)^{2}=\pi\left(R_{2}\right)^{2}=0, \quad\left[\pi(c), \pi\left(R_{1}\right)\right]=-\pi\left(R_{1}\right), \quad\left[\pi(c), \pi\left(R_{2}\right)\right]=\pi\left(R_{2}\right), \\
{\left[\pi\left(\Omega_{i}\right), \pi\left(R_{1}\right)\right]=\left[\pi\left(\Omega_{i}\right), \pi\left(R_{2}\right)\right]=0, \quad i=2, \ldots, n,} \\
{\left[\pi\left(R_{1}\right), \pi\left(R_{2}\right)\right]=\pi(\tilde{\Omega}),}
\end{gathered}
$$

where

$$
\tilde{\Omega}=\prod_{y \in B\left(\mathfrak{g}^{-1}\right)} \operatorname{ad} y\left(\prod_{x \in B\left(\mathfrak{g}^{1}\right)} x\right)
$$

is an element of $Z(\mathfrak{g})$. In this case $W_{\chi} \cong U\left(\mathfrak{g}^{e}\right)$.

\section{The case of $\mathfrak{g}=\mathfrak{g l}(n \mid n)$}

In this section, we outline the principal finite $W$-algebra for $\mathfrak{g l}(n \mid n)$.

Form: $(a \mid b)=\operatorname{str}(a b)$. We will use the following notations for some elementary matrices in $\mathfrak{g l}(n \mid n)$ : 


$$
\left(\begin{array}{ccccc|ccccc}
h_{1} & e_{1} & \ldots & \ldots & \ldots & y_{1} & y_{n+1} & \ldots & \ldots & \ldots \\
f_{1} & h_{2} & e_{2} & \ldots & \ldots & \mu_{1} & y_{2} & y_{n+2} & \ldots & \ldots \\
\ldots & \ldots & \ldots & \ldots & \ldots & \ldots & \ldots & \ldots & \ldots & \ldots \\
\ldots & \ldots & f_{n-2} & h_{n-1} & e_{n-1} & \ldots & \ldots & \mu_{n-2} & y_{n-1} & y_{2 n-1} \\
\ldots & \ldots & \ldots & f_{n-1} & h_{n} & \ldots & \ldots & \ldots & \mu_{n-1} & y_{n} \\
\hline x_{1} & x_{n+1} & \ldots & \ldots & \ldots & h_{n+1} & e_{n} & \ldots & \ldots & \ldots \\
\xi_{1} & x_{2} & x_{n+2} & \ldots & \ldots & g_{1} & h_{n+2} & e_{n+1} & \ldots & \ldots \\
\ldots & \ldots & \ldots & \ldots & \ldots & \ldots & \ldots & \ldots & \ldots & \ldots \\
\ldots & \ldots & \xi_{n-2} & x_{n-1} & x_{2 n-1} & \ldots & \ldots & g_{n-2} & h_{2 n-1} & e_{2 n-2} \\
\ldots & \ldots & \ldots & \xi_{n-1} & x_{n} & \ldots & \ldots & \ldots & g_{n-1} & h_{2 n}
\end{array}\right)
$$

Let $\mathfrak{s l}(2)=<e, h, f>$, where $e=e_{1}+e_{2}+\cdots e_{2 n-2}, f=\sum_{i=1}^{n-1} i(n-i)\left(f_{i}+g_{i}\right)$, $h=\operatorname{diag}(n-1, n-3, \cdots, 1-n \mid n-1, n-3, \cdots, 1-n)$. Note that $e$ is a regular nilpotent element. Let $z=\operatorname{diag}(1, \cdots, 1 \mid 1, \cdots, 1), c=\operatorname{diag}(1, \cdots, 1 \mid-1, \cdots,-1)$. Note that $\operatorname{dim}\left(\mathfrak{g}^{e}\right)=(2 n \mid 2 n)$. Explicitly, $\mathfrak{g}^{e}=\mathfrak{g}_{\overline{0}}^{e} \oplus \mathfrak{g}_{\overline{1}}^{e}$, where

$\mathfrak{g}_{0}^{e}=<\left(e_{1}+\cdots+e_{n-1}\right)^{i},\left(e_{n}+\cdots+e_{2 n-2}\right)^{i}, z, c>$, $\mathfrak{g}_{\overline{1}}^{e}=<\left(x_{1}+\cdots+x_{n}\right),\left(y_{1}+\cdots+y_{n}\right),\left(x_{n+1}+\cdots+x_{2 n-1}\right)^{i},\left(y_{n+1}+\cdots+y_{2 n-1}\right)^{i}>$, where $i=1, \ldots, n-1$, and the powers are considered in the corresponding $n \times n$ matrices.

Theorem 16.1. [PS1]. In the case when $\mathfrak{g}=\mathfrak{g l}(n \mid n)$, $\mathfrak{g}^{e}$ is isomorphic to the truncated Lie superalgebra of polynomial currents in $\mathfrak{g l}(1 \mid 1)$.

$$
\mathfrak{g l}(1 \mid 1)=\left\{\left(\begin{array}{l|l}
a_{11} & a_{12} \\
\hline a_{21} & a_{22}
\end{array}\right) \mid a_{i j} \in \mathbb{C}\right\}
$$

The isomorphism

$$
\varphi: \mathfrak{g}^{e} \longrightarrow \mathfrak{g r}(1 \mid 1) \otimes \mathbb{C}[t] /\left(t^{n}\right)
$$

is given as follows:

$$
\begin{array}{ll}
\varphi\left(\left(e_{1}+\cdots+e_{n-1}\right)^{i}\right)=E_{11} \otimes t^{i}, & \varphi\left(\left(e_{n}+\cdots+e_{2 n-2}\right)^{i}\right)=E_{22} \otimes t^{i}, \\
\varphi\left(\left(x_{n+1}+\cdots+x_{2 n-1}\right)^{i}\right)=E_{21} \otimes t^{i}, & \varphi\left(\left(y_{n+1}+\cdots+y_{2 n-1}\right)^{i}\right)=E_{12} \otimes t^{i},
\end{array}
$$$$
\text { for } i=1, \ldots, n-1 \text {, }
$$

$\varphi\left(\frac{z+c}{2}\right)=E_{11}, \varphi\left(\frac{z-c}{2}\right)=E_{22}, \varphi\left(x_{1}+\cdots+x_{n}\right)=E_{21}, \varphi\left(y_{1}+\cdots+y_{n}\right)=E_{12}$.

$h$ defines an even Dynkin $\mathbb{Z}$-grading of $\mathfrak{g l}(n \mid n)$ whose degrees on the elementary matrices are 


$$
\begin{gathered}
\left(\begin{array}{cccc|cccc}
0 & 2 & \cdots & 2 n-2 & 0 & 2 & \cdots & 2 n-2 \\
-2 & 0 & \cdots & 2 n-4 & -2 & 0 & \cdots & 2 n-4 \\
\cdots & \cdots & \cdots & \cdots & \cdots & \cdots & \cdots & \cdots \\
2-2 n & \cdots & \cdots & 0 & 2-2 n & \cdots & \cdots & 0 \\
\hline 0 & 2 & \cdots & 2 n-2 & 0 & 2 & \cdots & 2 n-2 \\
-2 & 0 & \cdots & 2 n-4 & -2 & 0 & \cdots & 2 n-4 \\
\cdots & \cdots & \cdots & \cdots & \cdots & \cdots & \cdots & \cdots \\
2-2 n & \cdots & \cdots & 0 & 2-2 n & \cdots & \cdots & 0
\end{array}\right) . \\
\mathfrak{m}=\bigoplus_{j=2}^{n} \mathfrak{g}_{2-2 j},
\end{gathered}
$$

$\mathfrak{m}$ is generated by $\xi_{i}, \mu_{i}, f_{i}, g_{i}$ for $i=1, \cdots, n-1$ Note that

$$
\chi\left(f_{i}\right)=-\chi\left(g_{i}\right)=1 ; \quad \chi\left(\mu_{i}\right)=\chi\left(\xi_{i}\right)=0, \text { for } i=1, \cdots, n-1 .
$$

Note that $W_{\chi}$ is generated by $2 n$ even elements and $2 n$ odd elements.

The generators of $W_{\chi}$ can be identified with elements of $\mathfrak{g}^{e}$ using the map

$$
P: W_{\chi} \longrightarrow S\left(\mathfrak{g}^{e}\right) \text {. }
$$

\section{The super-Yangian of $\mathfrak{g l}(m \mid n)$}

In this section, we outline the correspondence between finite $W$-algebras for $\mathfrak{g l}(m \mid n)$ and super-Yangians.

Recall that for a finite-dimensional semi-simple Lie algebra $\mathfrak{g}$, the Yangian of $\mathfrak{g}$ is an infinite-dimensional Hopf algebra $Y(\mathfrak{g})$. It is a deformation of the universal enveloping algebra of the Lie algebra of polynomial currents of $\mathfrak{g}[\mathrm{M}]$.

Definition 17.1. The super-Yangian $Y(\mathfrak{g l}(m \mid n))$ of $\mathfrak{g l}(m \mid n)$ is an associative unital superalgebra over $\mathbb{C}$ with a countable set of generators

$$
T_{i, j}^{(1)}, T_{i, j}^{(2)}, \ldots, \text { where } i, j=1, \ldots, m+n \text {. }
$$

The $\mathbb{Z}_{2}$-grading of the algebra $Y(\mathfrak{g l}(m \mid n))$ is defined as follows:

$$
p\left(T_{i, j}^{(n)}\right)=p(i)+p(j)
$$

To write down defining relation for the generators of $Y(\mathfrak{g l}(m \mid n))$ we employ the formal series in $Y\left(\mathfrak{g l}(m \mid n)\left[\left[u^{-1}\right]\right]\right.$ :

$$
T_{i, j}(u)=\delta_{i j} \cdot 1+T_{i, j}^{(1)} u^{-1}+T_{i, j}^{(2)} u^{-2}+\ldots
$$

For all indices $i, j, k, l$ we have the relations 


$$
\begin{aligned}
& (u-v)\left[T_{i, j}(u), T_{k, l}(v)\right]= \\
& (-1)^{p(i) p(k)+p(i) p(l)+p(k) p(l)}\left(\left(T_{k, j}(u) T_{i, l}(v)-T_{k, j}(v) T_{i, l}(u)\right),\right.
\end{aligned}
$$

where $v$ is a formal parameter independent of $u$, so that (2) is an equality in the algebra of formal Laurent series in $u^{-1}, v^{-1}$ with coefficients in $Y(\mathfrak{g r}(m \mid n))$.

The following Proposition follows from [BR].

Proposition 17.1. In the case when $\mathfrak{g}=\mathfrak{g l}(n \mid n)$, the corresponding principal finite $W$-algebra is isomorphic to the truncated super-Yangian $Y(\mathfrak{g l}(1 \mid 1)) /(n)$.

Remark 17.1. The principal finite $W$-algebras for $\mathfrak{g l}(m \mid n)$ associated to regular nilpotent elements were described as certain truncations of a shifted version of the super-Yangian $Y(\mathfrak{g l}(1 \mid 1))$ by J. Brown, J. Brundan and S. Goodwin in 2012 $[B B G]$. They also classified irreducible modules over principal finite $W$-algebras for $\mathfrak{g l}(m \mid n)$ by highest weight theory and proved that they are finite-dimensional.

In 2003 C. Briot and E. Ragoucy observed that certain finite W-algebras based on $\mathfrak{g l}(m \mid n)$ can be realized as truncations of the super-Yangian of $\mathfrak{g l}(m \mid n)$ [BR]. They also observed that finite $W$-algebras for $\mathfrak{g l}(m \mid n)$ associated to non-regular nilpotent elements are connected to higher rank super-Yangians.

\section{The case of $\mathfrak{g}=\mathbf{Q}(\mathbf{n})$}

In this section, we construct a complete set of generators of the principal finite $W$-algebra for $Q(n)$.

The queer Lie superalgebra is defined as follows

$$
Q(n):=\left\{\left(\begin{array}{l|l}
A & B \\
\hline B & A
\end{array}\right) \mid A, B \text { are } n \times n \text { matrices }\right\} .
$$

Let $\operatorname{otr}\left(\begin{array}{c|c}A & B \\ \hline B & A\end{array}\right)=\operatorname{tr} B$.

Remark 18.1. $Q(n)$ has one-dimensional center $\left\langle z>\right.$, where $z=1_{2 n}$. Let

$$
S Q(n)=\{X \in Q(n) \mid \text { otr } X=0\} .
$$

Note that the Lie superalgebra $\tilde{Q}(n):=S Q(n) /<z>$ is simple.

Let $e_{i, j}$ and $f_{i, j}$ be standard bases in $A$ and $B$ respectively:

$$
e_{i, j}=\left(\begin{array}{c|c}
E_{i j} & 0 \\
\hline 0 & E_{i j}
\end{array}\right), \quad f_{i, j}=\left(\begin{array}{c|c}
0 & E_{i j} \\
\hline E_{i j} & 0
\end{array}\right),
$$

where $E_{i j}$ are elementary $n \times n$ matrices. 
Note that $\mathfrak{g}=Q(n)$ admits an odd non-degenerate $\mathfrak{g}$-invariant super symmetric bilinear form

$$
(x \mid y):=\operatorname{otr}(x y) \text { for } x, y \in \mathfrak{g} .
$$

Therefore, we identify the coadjoint module $\mathfrak{g}^{*}$ with $\Pi(\mathfrak{g})$, where $\Pi$ is the functor changing the parity.

Let $\mathfrak{s l}(2)=<e, h, f>$, where

$$
e=\sum_{i=1}^{n-1} e_{i, i+1}, \quad h=\operatorname{diag}(n-1, n-3, \ldots, 3-n, 1-n), \quad f=\sum_{i=1}^{n-1} i(n-i) e_{i+1, i} .
$$

Note that $e$ is a regular nilpotent element, $h$ defines an even Dynkin $\mathbb{Z}$-grading of $\mathfrak{g}$ whose degrees on the elementary matrices are

$$
\left(\begin{array}{cccc|cccc}
0 & 2 & \cdots & 2 n-2 & 0 & 2 & \cdots & 2 n-2 \\
-2 & 0 & \cdots & 2 n-4 & -2 & 0 & \cdots & 2 n-4 \\
\cdots & \cdots & \cdots & \cdots & \cdots & \cdots & \cdots & \cdots \\
2-2 n & \cdots & \cdots & 0 & 2-2 n & \cdots & \cdots & 0 \\
\hline 0 & 2 & \cdots & 2 n-2 & 0 & 2 & \cdots & 2 n-2 \\
-2 & 0 & \cdots & 2 n-4 & -2 & 0 & \cdots & 2 n-4 \\
\cdots & \cdots & \cdots & \cdots & \cdots & \cdots & \cdots & \cdots \\
2-2 n & \cdots & \cdots & 0 & 2-2 n & \cdots & \cdots & 0
\end{array}\right) .
$$

Let $E=\sum_{i=1}^{n-1} f_{i, i+1}$. Since we have an isomorphism $\mathfrak{g}^{*} \cong \Pi(\mathfrak{g})$, an even regular nilpotent $\chi \in \mathfrak{g}^{*}$ can be defined by $\chi(x):=(x \mid E)$ for $x \in \mathfrak{g}$. Note that

$$
\mathfrak{g}^{E}=\left\{z, e, e^{2}, \ldots, e^{n-1} \mid H_{0}, H_{1}, \ldots, H_{n-1}\right\}, \quad \operatorname{dim}\left(\mathfrak{g}^{E}\right)=(n \mid n),
$$

where $H_{0}=\sum_{i=1}^{n}(-1)^{i-1} f_{i, i}, H_{1}=\sum_{i=1}^{n-1}(-1)^{i} f_{i, i+1}, \ldots, H_{n-1}=(-1)^{n-1} f_{1, n}$. Let

$$
\mathfrak{m}=\bigoplus_{j=2}^{n} \mathfrak{g}_{2-2 j}
$$

Note that $\mathfrak{m}$ is generated by $e_{i+1, i}$ and $f_{i+1, i}$, where $i=1, \ldots, n-1$, and

$$
\chi\left(e_{i+1, i}\right)=1, \quad \chi\left(e_{i+k, i}\right)=0 \text { if } k \geq 2, \quad \chi\left(f_{i+k, i}\right)=0 \text { if } k \geq 1 .
$$

The left ideal $I_{\chi}$ and $W_{\chi}$ are defined now as usual. $[\mathrm{S}]:$

A. Sergeev defined by induction the elements $e_{i, j}^{(m)}$ and $f_{i, j}^{(m)}$ belonging to $U(\mathfrak{g})$

$$
\begin{aligned}
& e_{i, j}^{(m)}=\sum_{k=1}^{n} e_{i, k} e_{k, j}^{(m-1)}+(-1)^{m+1} \sum_{k=1}^{n} f_{i, k} f_{k, j}^{(m-1)}, \\
& f_{i, j}^{(m)}=\sum_{k=1}^{n} e_{i, k} f_{k, j}^{(m-1)}+(-1)^{m+1} \sum_{k=1}^{n} f_{i, k} e_{k, j}^{(m-1)} .
\end{aligned}
$$


Then

$$
\begin{aligned}
& {\left[e_{i, j}, e_{k, l}^{(m)}\right]=\delta_{j, k} e_{i, l}^{(m)}-\delta_{i, l} e_{k, j}^{(m)}, \quad\left[e_{i, j}, f_{k, l}^{(m)}\right]=\delta_{j, k} f_{i, l}^{(m)}-\delta_{i, l} f_{k j}^{(m)},} \\
& {\left[f_{i, j}, e_{k, l}^{(m)}\right]=(-1)^{m+1} \delta_{j, k} f_{i, l}^{(m)}-\delta_{i, l} f_{k, j}^{(m)}, \quad\left[f_{i, j}, f_{k, l}^{(m)}\right]=(-1)^{m+1} \delta_{j, k} e_{i, l}^{(m)}+\delta_{i, l} e_{k, j}^{(m)} .}
\end{aligned}
$$

Proposition 18.1. A. Sergeev [S].

The elements $\sum_{i=1}^{n} e_{i, i}^{(2 m+1)}$ generate $Z(\mathfrak{g})$.

Theorem 18.1. [PS2]. $\pi\left(e_{n, 1}^{(m)}\right)$ and $\pi\left(f_{n, 1}^{(m)}\right)$ are Whittaker vectors.

$W_{\chi}$ has $n$ even generators $\pi\left(e_{n, 1}^{(n+k-1)}\right)$ and $n$ odd generators $\pi\left(f_{n, 1}^{(n+k-1)}\right)$, for $k=$ $1, \ldots, n$. The generators of $W_{\chi}$ can be identified with elements of $\mathfrak{g}^{E}$ :

$$
\begin{gathered}
\pi\left(e_{n, 1}^{(n)}\right) \stackrel{P}{\longrightarrow} z, \quad \pi\left(e_{n, 1}^{(n+k-1)}\right) \stackrel{P}{\longrightarrow} e^{k-1}, \quad k=2, \ldots, n, \\
\pi\left(f_{n, 1}^{(n+k-1)}\right) \stackrel{P}{\longrightarrow} H_{k-1}, \quad k=1, \ldots, n .
\end{gathered}
$$

Corollary 18.1. The natural homomorphism $U(\mathfrak{g})^{a d \mathfrak{m}} \longrightarrow W_{\chi}$ is surjective.

Let $\mathfrak{p}:=\oplus_{j \geq 0} \mathfrak{g}_{j}$. Let $\mathfrak{f}=<e_{i, i}, f_{i, i} \mid i=1, \ldots, n>$, and let $\vartheta: U(\mathfrak{p}) \longrightarrow U(\mathfrak{f})$ be the Harish-Chandra homomorphism.

Denote

$$
x_{i}=e_{i, i}, \quad \xi_{i}=(-1)^{i+1} f_{i, i} .
$$

Theorem 18.2. [PS2]. The Harish-Chandra homomorphism is injective.

Under the Harish-Chandra homomorphism:

$$
\begin{aligned}
& \vartheta\left(\pi\left(e_{n, 1}^{(n+k-1)}\right)\right)=\left[\sum_{i_{1} \geq i_{2} \geq \ldots \geq i_{k}}\left(x_{i_{1}}+(-1)^{k+1} \xi_{i_{1}}\right) \ldots\left(x_{i_{k-1}}-\xi_{i_{k-1}}\right)\left(x_{i_{k}}+\xi_{i_{k}}\right)\right]_{\text {even }}, \\
& \vartheta\left(\pi\left(f_{n, 1}^{(n+k-1)}\right)\right)=\left[\sum_{i_{1} \geq i_{2} \geq \ldots \geq i_{k}}\left(x_{i_{1}}+(-1)^{k+1} \xi_{i_{1}}\right) \ldots\left(x_{i_{k-1}}-\xi_{i_{k-1}}\right)\left(x_{i_{k}}+\xi_{i_{k}}\right)\right]_{\text {odd }} .
\end{aligned}
$$

Theorem 18.3. [PS2].

$$
\pi\left(e_{n, 1}^{(n+1)}\right)=\pi\left(\frac{1}{2} \sum_{i=1}^{n} e_{i, i}^{2}+\sum_{i=1}^{n-1} e_{i, i+1}+\sum_{i<j}(-1)^{i-j} f_{i, i} f_{j, j}+\frac{1}{2} z^{2}-z\right)
$$

One can define odd generators $\Phi_{0}, \ldots, \Phi_{n-1}$ of $W_{\chi}$ as follows: 


$$
\begin{aligned}
\Phi_{0} & =\pi\left(f_{n, 1}^{(n)}\right)=\pi\left(H_{0}\right), \\
\Phi_{1} & =\left[\pi\left(e_{n, 1}^{(n+1)}\right), \Phi_{0}\right], \\
\cdots & \\
\Phi_{n-1} & =\left[\pi\left(e_{n, 1}^{(n+1)}\right), \Phi_{n-2}\right] .
\end{aligned}
$$

Then

$$
\begin{gathered}
{\left[\Phi_{m}, \Phi_{p}\right]=0, \text { if } m+p \text { is odd, }} \\
{\left[\Phi_{m}, \Phi_{p}\right] \in Z(\mathfrak{g}), \text { if } m+p \text { is even } .}
\end{gathered}
$$

Lemma 18.1. For odd $m$ and $p$ we have

$$
\left[\pi\left(e_{n, 1}^{(n+m)}\right), \pi\left(e_{n, 1}^{(n+p)}\right)\right]=0 .
$$

We set

$$
\begin{array}{cc}
z_{i}=\pi\left(e_{n, 1}^{(n+i)}\right) & \text { for odd } i \\
z_{i}=\left[\Phi_{0}, \Phi_{i}\right] & \text { for even } i
\end{array}
$$

Theorem 18.4. [PS2]. Elements $z_{0}, \ldots, z_{n-1}$ are algebraically independent in $W_{\chi}$. Together with $\Phi_{0}, \ldots, \Phi_{n-1}$ they form a complete set of generators in $W_{\chi}$.

Example 18.1. $n=2, \mathfrak{g}=Q(2)$.

Let

$$
\begin{gathered}
e=e_{1,2}, \quad h=\operatorname{diag}(1,-1), \quad f=e_{2,1}, \quad z=e_{1,1}+e_{2,2} \\
E=f_{1,2}, \quad H_{0}=f_{1,1}-f_{2,2}, \quad H_{1}=-f_{1,2} .
\end{gathered}
$$

Then

$$
\mathfrak{g}^{E}=\left\{z, e \mid H_{0}, H_{1}\right\}, \quad \operatorname{dim}\left(\mathfrak{g}^{E}\right)=(2 \mid 2) .
$$

According to Theorem 7, $W_{\chi}$ has 2 even generators: $\pi\left(e_{2,1}^{(2)}\right), \pi\left(e_{2,1}^{(3)}\right)$, and 2 odd generators: $\pi\left(f_{2,1}^{(2)}\right), \pi\left(f_{2,1}^{(3)}\right)$. Let

$$
\begin{gathered}
\Phi_{0}=\pi\left(f_{2,1}^{(2)}\right)=\pi\left(H_{0}\right), \\
\Phi_{1}=\left[\pi\left(e_{2,1}^{(3)}\right), \Phi_{0}\right]=2 \pi\left(f_{2,1}^{(3)}\right)=2 \pi\left(-f_{1,2}+f_{1,1} e_{2,2}+f_{2,2} e_{1,1}\right) .
\end{gathered}
$$

Let

$$
\begin{gathered}
z_{0}=2 \pi\left(e_{2,1}^{(2)}\right)=2 \pi(z), \\
z_{1}=\pi\left(e_{2,1}^{(3)}\right)=\pi\left(e_{1,2}+e_{1,1}^{2}+e_{2,2}^{2}+e_{1,1} e_{2,2}-e_{1,1}-e_{2,2}-f_{1,1} f_{2,2}\right) .
\end{gathered}
$$

The nonzero commutation relations between the generators of $W_{\chi}$ are as follows: 


$$
\begin{gathered}
{\left[z_{1}, \Phi_{0}\right]=\Phi_{1}, \quad\left[z_{1}, \Phi_{1}\right]=4 z_{1} \Phi_{0}-2 \Phi_{1}-z_{0}^{2} \Phi_{0}+2 z_{0} \Phi_{0}} \\
{\left[\Phi_{0}, \Phi_{0}\right]=z_{0}, \quad\left[\Phi_{1}, \Phi_{1}\right]=4 \Phi_{1} \Phi_{0}-4 z_{0} z_{1}+\frac{3}{4} z_{0}^{3}-z_{0}^{2}}
\end{gathered}
$$

Note that

$$
\left[\Phi_{1}, \Phi_{1}\right]=\frac{1}{3} \pi\left(-8\left(e_{1,1}^{(3)}+e_{2,2}^{(3)}\right)+2 z^{3}+4 z^{2}\right) .
$$

Hence by Proposition 5

$$
\left[\Phi_{1}, \Phi_{1}\right] \in \pi(Z(\mathfrak{g}))
$$

Remark 18.2. $L$. Zhao studied finite $W$-algebras for $\mathfrak{g}=Q(n)[Z]$. He proved that the definition of the finite $W$-algebra is independent of the choices of the isotropic subspaces $\mathfrak{l}$ and the good $\mathbb{Z}$-gradings. He also established a Skryabin type equivalence between the category of $W_{\chi}$-modules and a category of certain $\mathfrak{g}$-modules.

Conjecture 18.1. [PS2]. Irreducible modules over the principal finite $W$-algebra for $Q(n)$ are finite-dimensional.

Conjecture 18.2. [PS2]. In the case when $\operatorname{dim}\left(\mathfrak{g}_{-1}\right)_{\overline{1}}$ is even, it is possible to find a set of generators of the principal finite $W$-algebra for $\mathfrak{g}$ such that even generators commute, and the commutators of odd generators are in $\pi(Z(\mathfrak{g}))$.

\section{The super-Yangian of $\mathbf{Q}(\mathbf{n})$}

In this section, we describe the principal finite $W$-algebra for $Q(n)$ as a factor algebra of the super-Yangian of $Q(1)$.

The super-Yangian $Y(Q(n))$ was studied by M. Nazarov and A. Sergeev [N, NS]. Note that $Y(Q(n))$ is the associative unital superalgebra over $\mathbb{C}$ with the countable set of generators

$$
T_{i, j}^{(m)}, \text { where } m=1,2, \ldots \text { and } i, j= \pm 1, \pm 2, \ldots, \pm n .
$$

The $\mathbb{Z}_{2}$-grading of the algebra $Y(Q(n))$ is defined as follows:

$$
p\left(T_{i, j}^{(m)}\right)=p(i)+p(j), \text { where } p(i)=0 \text { if } i>0 \text { and } p(i)=1 \text { if } i<0 .
$$

To describe defining relations for the generators of $Y(Q(n))$ we employ the formal series in $Y(Q(n))\left[\left[u^{-1}\right]\right]$ :

$$
T_{i, j}(u)=\delta_{i j} \cdot 1+T_{i, j}^{(1)} u^{-1}+T_{i, j}^{(2)} u^{-2}+\ldots
$$


For all indices $i, j, k, l$ we have the relations

$$
\begin{aligned}
& \left(u^{2}-v^{2}\right)\left[T_{i, j}(u), T_{k, l}(v)\right] \cdot(-1)^{p(i) p(k)+p(i) p(l)+p(k) p(l)} \\
& \quad=(u+v)\left(T_{k, j}(u) T_{i, l}(v)-T_{k, j}(v) T_{i, l}(u)\right) \\
& -(u-v)\left(T_{-k, j}(u) T_{-i, l}(v)-T_{k,-j}(v) T_{i,-l}(u)\right) \cdot(-1)^{p(k)+p(l),}
\end{aligned}
$$

where $v$ is a formal parameter independent of $u$, so that (3) is an equality in the algebra of formal Laurent series in $u^{-1}, v^{-1}$ with coefficients in $Y(Q(n))$. We also have the relations

$$
T_{i, j}(-u)=T_{-i,-j}(u) .
$$

Relations (3) and (4) are equivalent to the following defining relations:

$$
\begin{aligned}
& \left(\left[T_{i, j}^{(m+1)}, T_{k, l}^{(r-1)}\right]-\left[T_{i, j}^{(m-1)}, T_{k, l}^{(r+1)}\right]\right) \cdot(-1)^{p(i) p(k)+p(i) p(l)+p(k) p(l)}= \\
& T_{k, j}^{(m)} T_{i, l}^{(r-1)}+T_{k, j}^{(m-1)} T_{i, l}^{(r)}-T_{k, j}^{(r-1)} T_{i, l}^{(m)}-T_{k, j}^{(r)} T_{i, l}^{(m-1)} \\
& +(-1)^{p(k)+p(l)}\left(-T_{-k, j}^{(m)} T_{-i, l}^{(r-1)}+T_{-k, j}^{(m-1)} T_{-i, l}^{(r)}+T_{k,-j}^{(r-1)} T_{i,-l}^{(m)}-T_{k,-j}^{(r)} T_{i,-l}^{(m-1)}\right), \\
& T_{-i,-j}^{(m)}=(-1)^{m} T_{i, j}^{(m)}
\end{aligned}
$$

where $m, r=1, \ldots$ and $T_{i j}^{(0)}=\delta_{i j}$.

Theorem 19.1. [PS2]. There exists a surjective homomorphism:

$$
\varphi: Y(Q(1)) \longrightarrow W_{\chi},
$$

defined as follows:

$$
\varphi\left(T_{1,1}^{(k)}\right)=(-1)^{k} \pi\left(e_{n, 1}^{(n+k-1)}\right), \quad \varphi\left(T_{-1,1}^{(k)}\right)=(-1)^{k} \pi\left(f_{n, 1}^{(n+k-1)}\right) \text { for } k=1,2, \ldots
$$

\section{The case of $\mathfrak{g}=\Gamma\left(\sigma_{1}, \sigma_{2}, \sigma_{3}\right)$}

In this section, we describe the principal finite $W$-algebra for the exceptional Lie superalgebra $\Gamma\left(\sigma_{1}, \sigma_{2}, \sigma_{3}\right)$ in terms of generators and relations. We follow the construction of this Lie superalgebra given by M. Scheunert [Sch].

Let $\sigma_{1}, \sigma_{2}, \sigma_{3}$ be complex numbers such that $\sigma_{1}+\sigma_{2}+\sigma_{3}=0$. The family of Lie superalgebras $\Gamma\left(\sigma_{1}, \sigma_{2}, \sigma_{3}\right)$ is defined as follows:

$$
\begin{gathered}
\Gamma\left(\sigma_{1}, \sigma_{2}, \sigma_{3}\right)=\Gamma_{\overline{0}} \oplus \Gamma_{\overline{1}}, \text { where } \\
\Gamma_{\overline{0}}=\mathfrak{s l}(2)_{\overline{1}} \oplus \mathfrak{s l}(2)_{\overline{2}} \oplus \mathfrak{s l}(2)_{\overline{3}}, \quad \Gamma_{\overline{1}}=V_{1} \otimes V_{2} \otimes V_{3},
\end{gathered}
$$

where $V_{i}$ is the standard $\mathfrak{s l}(2)_{i}$-module for $i=1,2,3$. 
Let $\mathfrak{s l}(2)_{i}=<X_{i}, H_{i}, Y_{i}>$, where $\left\{X_{i}, H_{i}, Y_{i}\right\}$ is the standard basis in each $\mathfrak{s l}(2)_{i}$ for $i=1,2,3$. Let $V_{i}=<e_{i}, f_{i}>$ and let $P_{i}: V_{i} \times V_{i} \rightarrow \mathfrak{s l}(2)_{i}$ be the $\mathfrak{s l}(2)_{i}$-invariant bilinear mapping given by

$$
P_{i}\left(e_{i}, e_{i}\right)=2 X_{i}, \quad P_{i}\left(f_{i}, f_{i}\right)=-2 Y_{i}, \quad P_{i}\left(e_{i}, f_{i}\right)=P_{i}\left(f_{i}, e_{i}\right)=-H_{i} .
$$

Let $\psi_{i}$ be a non-degenerate skew-symmetric form on $V_{i}$ :

$$
\psi_{i}\left(e_{i}, f_{i}\right)=-\psi_{i}\left(f_{i}, e_{i}\right)=1 .
$$

Note that $\left[\Gamma_{\overline{0}}, \Gamma_{\overline{1}}\right]$ is the tensor product of the standard representations of $\mathfrak{s l}(2)_{i}$ in $V_{i}$, and $\left[\Gamma_{\overline{1}}, \Gamma_{\overline{1}}\right]$ is given by the formula

$$
\begin{gathered}
{\left[x_{1} \otimes x_{2} \otimes x_{3}, y_{1} \otimes y_{2} \otimes y_{3}\right]=\sigma_{1} \psi_{2}\left(x_{2}, y_{2}\right) \psi_{3}\left(x_{3}, y_{3}\right) P_{1}\left(x_{1}, y_{1}\right)+} \\
\sigma_{2} \psi_{1}\left(x_{1}, y_{1}\right) \psi_{3}\left(x_{3}, y_{3}\right) P_{2}\left(x_{2}, y_{2}\right)+\sigma_{3} \psi_{1}\left(x_{1}, y_{1}\right) \psi_{2}\left(x_{2}, y_{2}\right) P_{3}\left(x_{3}, y_{3}\right),
\end{gathered}
$$

where $x_{i}, y_{i} \in V_{i}, i=1,2,3$.

Remark 20.1. The superalgebra $\Gamma\left(\sigma_{1}, \sigma_{2}, \sigma_{3}\right)$ is simple if and only if $\sigma_{i} \neq 0$ for $i=1,2,3$. $\Gamma\left(\sigma_{1}, \sigma_{2}, \sigma_{3}\right) \cong \Gamma\left(\sigma_{1}^{\prime}, \sigma_{2}^{\prime}, \sigma_{3}^{\prime}\right)$ if and only if the sets $\left\{\sigma_{i}^{\prime}\right\}$ and $\left\{\sigma_{i}\right\}$ are obtained from each other by a permutation and multiplication of all elements of one set by a nonzero complex number (see [Sch]). Thus $\Gamma\left(\sigma_{1}, \sigma_{2}, \sigma_{3}\right)$ is a one-parameter family of deformations of osp $(4 \mid 2)$. Note that $\Gamma(1,-1-\alpha, \alpha) \cong$ $D(2,1 ; \alpha)$, where $\alpha \neq 0,-1$ (see $[K])$.

We consider the non-degenerate invariant symmetric bilinear form on $\mathfrak{g}$ given as follows:

$$
\begin{gathered}
\left(X_{i}, Y_{i}\right)=\frac{1}{\sigma_{i}}, \quad\left(H_{i}, H_{i}\right)=\frac{2}{\sigma_{i}}, \\
\left(e_{1} \otimes e_{2} \otimes e_{3}, f_{1} \otimes f_{2} \otimes f_{3}\right)=-2, \quad\left(e_{1} \otimes e_{2} \otimes f_{3}, f_{1} \otimes f_{2} \otimes e_{3}\right)=2, \\
\left(e_{1} \otimes f_{2} \otimes e_{3}, f_{1} \otimes e_{2} \otimes f_{3}\right)=2, \quad\left(f_{1} \otimes e_{2} \otimes e_{3}, e_{1} \otimes f_{2} \otimes f_{3}\right)=2 .
\end{gathered}
$$

Let $\mathfrak{s l}(2)=<e, h, f>$, where

$$
e=X_{1}+X_{2}+X_{3}, \quad h=H_{1}+H_{2}+H_{3}, \quad f=Y_{1}+Y_{2}+Y_{3} .
$$

Then $e$ is a regular nilpotent element, $h$ defines a Dynkin $\mathbb{Z}$-grading of $\mathfrak{g}$ :

$$
\begin{gathered}
\mathfrak{g}=\oplus_{j=-3}^{3} \mathfrak{g}_{j}, \text { where } \\
\mathfrak{g}_{3}=<e_{1} \otimes e_{2} \otimes e_{3}>, \quad \mathfrak{g}_{2}=<X_{1}, X_{2}, X_{3}>, \\
\mathfrak{g}_{1}=<e_{1} \otimes e_{2} \otimes f_{3}, e_{1} \otimes f_{2} \otimes e_{3}, f_{1} \otimes e_{2} \otimes e_{3}>, \\
\mathfrak{g}_{0}=<H_{1}, H_{2}, H_{3}>, \quad \mathfrak{g}_{-1}=<e_{1} \otimes f_{2} \otimes f_{3}, f_{1} \otimes e_{2} \otimes f_{3}, e_{1} \otimes e_{2} \otimes f_{3}>, \\
\mathfrak{g}_{-2}=<Y_{1}, Y_{2}, Y_{3}>, \quad \mathfrak{g}_{-3}=<f_{1} \otimes f_{2} \otimes f_{3}>.
\end{gathered}
$$

Note that $\operatorname{dim}\left(\mathfrak{g}^{e}\right)=(3 \mid 3)$. Explicitly,

$\left(\mathfrak{g}^{e}\right)_{\overline{0}}=<X_{1}, X_{2}, X_{3}>$,

$\left(\mathfrak{g}^{e}\right)_{\overline{1}}=<e_{1} \otimes f_{2} \otimes e_{3}-e_{1} \otimes e_{2} \otimes f_{3}, f_{1} \otimes e_{2} \otimes e_{3}-e_{1} \otimes e_{2} \otimes f_{3}, e_{1} \otimes e_{2} \otimes e_{3}>$. 
In $\mathfrak{g}$ we consider a nilpotent subalgebra $\mathfrak{m}=\mathfrak{g}_{-3} \oplus \mathfrak{g}_{-2} \oplus \mathfrak{l}$, where $\mathfrak{l}$ is a onedimensional Lagrangian subspace of $\mathfrak{g}_{-1}: \mathfrak{l}=<e_{1} \otimes f_{2} \otimes f_{3}>$. Note that $\mathfrak{m}$ is generated by $Y_{1}, Y_{2}, Y_{3}$ and $e_{1} \otimes f_{2} \otimes f_{3}$. We have that

$$
\chi\left(Y_{i}\right)=\frac{1}{\sigma_{i}}, \quad \chi\left(e_{1} \otimes f_{2} \otimes f_{3}\right)=0 .
$$

Let $\theta=f_{1} \otimes e_{2} \otimes f_{3}-f_{1} \otimes f_{2} \otimes e_{3}$. Then $\theta \in \mathfrak{g}_{-1} \cap \mathfrak{l}^{\perp}, \pi(\theta) \in W_{\chi}, \quad \pi(\theta)^{2}=-2$.

Even generators of $W_{\chi}$ are:

$C_{1}=\pi\left(2 X_{1}+\sigma_{1}\left(\frac{1}{2} H_{1}^{2}-H_{1}\right)\right)$,

$C_{2}=\pi\left(2 X_{2}+\frac{1}{2} \sigma_{2} H_{2}^{2}+\left(f_{1} \otimes e_{2} \otimes f_{3}\right)\left(e_{1} \otimes e_{2} \otimes f_{3}\right)\right)$,

$C_{3}=\pi\left(2 X_{3}+\frac{1}{2} \sigma_{3} H_{3}^{2}+\left(f_{1} \otimes f_{2} \otimes e_{3}\right)\left(e_{1} \otimes f_{2} \otimes e_{3}\right)\right)$.

Odd generators of $W_{\chi}$ are

$R_{1}=\pi\left(2\left(e_{1} \otimes f_{2} \otimes e_{3}-e_{1} \otimes e_{2} \otimes f_{3}\right)+\sigma_{1} H_{1}\left(f_{1} \otimes e_{2} \otimes f_{3}-f_{1} \otimes f_{2} \otimes e_{3}\right)\right)$,

$R_{2}=\pi\left(2\left(f_{1} \otimes e_{2} \otimes e_{3}-e_{1} \otimes e_{2} \otimes f_{3}\right)\right.$

$\left.+\left(\sigma_{1} H_{1}-\sigma_{3} H_{3}\right)\left(f_{1} \otimes e_{2} \otimes f_{3}\right)-\sigma_{2} H_{2}\left(f_{1} \otimes f_{2} \otimes e_{3}\right)\right)$,

$R_{3}=\pi\left(4\left(e_{1} \otimes e_{2} \otimes e_{3}\right)-\sigma_{1} H_{1} R_{2}-4 \sigma_{1}\left(f_{1} \otimes e_{2} \otimes f_{3}\right) X_{1}\right.$

$\left.-2\left(\sigma_{1} H_{1}\left(e_{1} \otimes e_{2} \otimes f_{3}\right)+\sigma_{2} H_{2}\left(e_{1} \otimes f_{2} \otimes e_{3}\right)+\sigma_{3} H_{3}\left(e_{1} \otimes e_{2} \otimes f_{3}\right)\right)\right)$,

and $\pi(\theta)$. Note that the generators $C_{i}$ and $R_{i}$ for $i=1,2,3$ can be identified with the corresponding elements of $\mathfrak{g}^{e}$ under the mapping $P$ (see Remark 8 ). The quadratic Casimir element of $\mathfrak{g}$ is

$\Omega=\Sigma_{i=1}^{3}\left(\frac{\sigma_{i}}{2} H_{i}^{2}+2 X_{i}\right)-\left(e_{1} \otimes e_{2} \otimes f_{3}\right)\left(f_{1} \otimes f_{2} \otimes e_{3}\right)-\left(e_{1} \otimes f_{2} \otimes e_{3}\right)\left(f_{1} \otimes e_{2} \otimes f_{3}\right)$.

Hence

$$
\pi(\Omega)=C_{1}+C_{2}+C_{3}-\frac{1}{2} R_{1} \pi(\theta)
$$

Theorem 20.1. [P]. The principal finite $W$-algebra $W_{\chi}$ is generated by even elements $\pi(\Omega), C_{1}$ and $C_{2}$, and odd element $\pi(\theta)$. The relations are

$$
\begin{gathered}
{\left[C_{1}, C_{2}\right]=0, \quad\left[\pi(\theta), C_{i}\right]=R_{i} \mp \frac{\sigma_{i}}{2} \pi(\theta), \quad i=1,2,} \\
{\left[C_{2}, R_{1}\right]=-\frac{\sigma_{2}}{2} R_{1}+R_{3}, \quad\left[C_{1}, R_{2}\right]=\frac{\sigma_{1}}{2} R_{2}+R_{3},} \\
{\left[R_{i}, R_{i}\right]=8 \sigma_{i} C_{i}-2 \sigma_{i} R_{i} \pi(\theta), \quad i=1,2,} \\
{\left[R_{1}, R_{2}\right]=-4\left(\sigma_{1} C_{2}+\sigma_{2} C_{1}+\sigma_{3} \pi(\Omega)\right)+\left(\sigma_{1} R_{2}+\sigma_{2} R_{1}\right) \pi(\theta),} \\
{\left[R_{i}, \pi(\theta)\right]=\mp 2 \sigma_{i}, \quad i=1,2, \quad[\pi(\theta), \pi(\theta)]=-4,} \\
{[\pi(\Omega), \pi(\theta)]=0, \quad\left[\pi(\Omega), C_{i}\right]=0, i=1,2, \quad\left[\pi(\Omega), R_{i}\right]=0, i=1,2,3 .}
\end{gathered}
$$




\section{The case of $\mathfrak{g}=\mathfrak{v} \mathfrak{s p}(\mathbf{1} \mid \mathbf{2})$}

In this section, we describe the principal finite $W$-algebra for $\mathfrak{v} \mathfrak{s p}(1 \mid 2)$.

Form: $(a \mid b)=\frac{1}{2} \operatorname{str}(a b)$

$\mathfrak{g}=<e, f, h \mid \theta, r>$, where

$$
\begin{gathered}
e=\left(\begin{array}{l|ll}
0 & 0 & 0 \\
\hline 0 & 0 & 1 \\
0 & 0 & 0
\end{array}\right), f=\left(\begin{array}{l|ll}
0 & 0 & 0 \\
\hline 0 & 0 & 0 \\
0 & 1 & 0
\end{array}\right), h=\left(\begin{array}{c|cc}
0 & 0 & 0 \\
\hline 0 & 1 & 0 \\
0 & 0 & -1
\end{array}\right), \\
\theta=\left(\begin{array}{c|cc}
0 & 1 & 0 \\
\hline 0 & 0 & 0 \\
-1 & 0 & 0
\end{array}\right), r=\left(\begin{array}{l|ll}
0 & 0 & 1 \\
\hline 1 & 0 & 0 \\
0 & 0 & 0
\end{array}\right) .
\end{gathered}
$$

Note that $e$ is a regular nilpotent element, and $h$ defines a $\mathbb{Z}$-grading of $\mathfrak{g}$ :

$$
\mathfrak{g}=\mathfrak{g}_{-2} \oplus \mathfrak{g}_{-1} \oplus \mathfrak{g}_{0} \oplus \mathfrak{g}_{1} \oplus \mathfrak{g}_{2},
$$

where

$$
\begin{aligned}
& \mathfrak{g}_{-2}=<f>, \quad \mathfrak{g}_{-1}=<\theta>, \quad \mathfrak{g}_{0}=<h>, \quad \mathfrak{g}_{1}=<r>, \quad \mathfrak{g}_{2}=<e>. \\
& \mathfrak{m}=\mathfrak{g}_{-2}=<f>, \quad \chi(f)=-\frac{1}{2} . \\
& \mathfrak{g}^{e}=<e \mid r>, \quad \operatorname{dim} \mathfrak{g}^{e}=(1 \mid 1) .
\end{aligned}
$$

Note that $\pi(\theta) \in W_{\chi}$, and $\pi(\theta)^{2}=\frac{1}{2}$.

Even generator of $W_{\chi}$ is $\pi(\Omega)$, where $\Omega=2 e+h-h^{2}+2 r \theta$ is the Casimir element of $\mathfrak{g}$.

Odd generators of $W_{\chi}$ are $R=\pi(r-h \theta)$ and $\pi(\theta)$.

Note that generators $\pi(\Omega)$ and $R$ are identified with elements of $\mathfrak{g}^{e}$ :

$$
\begin{gathered}
\frac{1}{2} \pi(\Omega) \stackrel{P}{\longrightarrow} e, \\
R \stackrel{P}{\longrightarrow} r .
\end{gathered}
$$

Theorem 21.1. The principal finite $W$-algebra $W_{\chi}$ is generated by $\pi(\Omega)$ and two odd generators: $\pi(\theta)$ and $R$. The defining relations are

$$
\begin{gathered}
{[\pi(\Omega), R]=[\pi(\Omega), \pi(\theta)]=0,} \\
{[R, R]=\pi(\Omega), \quad[R, \pi(\theta)]=-\frac{1}{2}, \quad[\pi(\theta), \pi(\theta)]=1 .}
\end{gathered}
$$




\section{The case of $\mathfrak{g}=\mathfrak{v} \mathfrak{s p}(1 \mid 2 n)$}

In this section, we present partial results for the principal finite $W$-algebra for $\mathfrak{v} \mathfrak{s p}(1 \mid 2 n)$, where $n \geq 2$, and make a conjecture for this case.

Form: $(a \mid b)=-\operatorname{str}(a b)$.

We will use the following notations for some elementary matrices in $\mathfrak{v} \mathfrak{p}(1 \mid 2 n)$ :

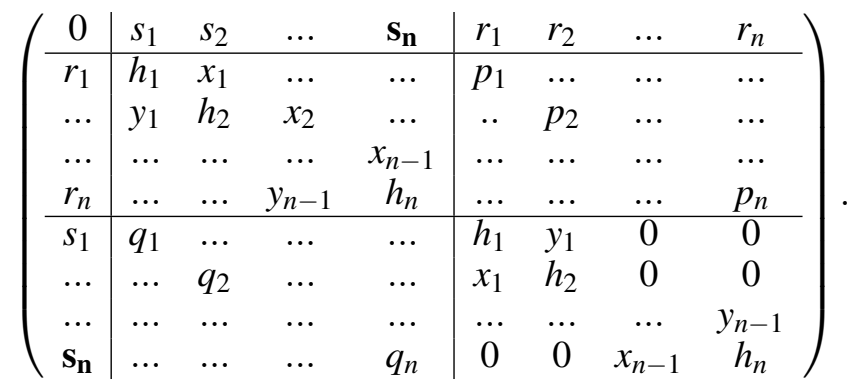

Let $\mathfrak{s l}(2)=<e, h, f>$, where $e=\left(x_{1}+\ldots x_{n-1}\right)+p_{n}$,

$h=\operatorname{diag}(0 \mid 2 n-1,2 n-3, \ldots, 3,1 ;-2 n+1,-2 n+3, \ldots,-3,-1)$,

$f=\left(\sum_{k=1}^{n-1} k(2 n-k) y_{k}\right)+n^{2} q_{n}$.

Note that $e$ is a regular nilpotent element, and $h$ defines a Dynkin $\mathbb{Z}$-grading of $\mathfrak{g}$ whose degrees on the elementary matrices are

$\left(\begin{array}{c|cccc|cccc}0 & -2 n+1 & \ldots & -3 & -\mathbf{1} & 2 n-1 & \ldots & 3 & 1 \\ \hline 2 n-1 & 0 & 2 & \ldots & \ldots & 4 n-2 & \ldots & 2 n+2 & 2 n \\ \ldots & -2 & 0 & 2 & \ldots & 4 n-4 & \ldots & 2 n & 2 n-2 \\ 3 & \ldots & \ldots & 0 & 2 & \ldots & \ldots & 6 & 4 \\ 1 & \ldots & -4 & -2 & 0 & 2 n & \ldots & 4 & 2 \\ \hline-2 n+1 & -4 n+2 & \ldots & -2 n-2 & -2 n & 0 & -2 & 0 & 0 \\ \ldots & -4 n+4 & \ldots & -2 n & -2 n+2 & 2 & 0 & -2 & 0 \\ -3 & \ldots & \ldots & -6 & -4 & \ldots & \ldots & 0 & -2 \\ -1 & -2 n & \ldots & -4 & -2 & \ldots & \ldots & 2 & 0\end{array}\right)$.

Note that $\operatorname{dim}^{e}=(n \mid 1),\left(\mathfrak{g}^{e}\right)_{\overline{1}}=<r_{1}>, \mathfrak{g}_{-1}=<\theta>$, where $\theta=s_{n}, \operatorname{dim}_{-1}=1$.

Note that $\mathfrak{m}=\oplus_{j \leq-2} \mathfrak{g}_{j}$, and $\mathfrak{g}_{-2}=<y_{i}, q_{n}>, i=1, \ldots, n-1$.

$\mathfrak{m}$ is generated by $y_{i}, q_{n}, \chi\left(y_{i}\right)=2$, for $i=1, \ldots, n-1$, and $\chi\left(q_{n}\right)=1$.

$\pi(\theta) \in W_{\chi}, \pi(\theta)^{2}=-1$.

Conjecture 22.1. [P]. The principal finite $W$-algebra $W_{\chi}$ is generated by the first $n$ Casimir elements in $\pi(Z(\mathfrak{g}))$ and odd elements $\pi(\theta)$ and $R$, where $R$ is induced 
by $r_{1}$ so that

$$
[R, R] \in \pi(Z(\mathfrak{g})), \quad[R, \pi(\theta)] \in \pi(Z(\mathfrak{g})), \quad[\pi(\theta), \pi(\theta)]=-2 .
$$

\section{Acknowledgments}

The author would like to thank V. Serganova for very helpful discussions.

\section{References}

[A] Eiichi Abe, Hopf Algebras, Cambridge Tracts in Mathematics 74, Cambridge University Press (2004).

[B] J. Balog, L. Fehér and L. O'Raifeartaigh, Toda theory and W-algebra from a gauged WZNW point of view, Ann. Phys. 203 (1990) 76-136.

[BR] C. Briot, E. Ragoucy, $W$-superalgebras as truncations of super-Yangians, J. Phys. A 36 (2003), no. 4, 1057-1081.

[BG] J. Brundan and S. Goodwin, Good grading polytopes. Proc. London Math. Soc. 94 (2007) 155-180.

[BBG] J. Brown, J. Brundan, S. Goodwin, Principal $W$-algebras for $G L(m \mid n)$, arXiv:1205.0992.

[BK1] J. Brundan and A. Kleshchev Shifted Yangians and finite $W$-algebras, Adv. Math. 200 (2006) 136-195.

[BK2] J. Brundan and A. Kleshchev Representations of shifted Yangians and finite W-algebras, Mem. Amer. Math. Soc. 196 (2008), no. 918, 107 pp.

[C] R. Carter, Finite groups of Lie type: Conjugacy classes and complex characters. Pure and Applied Math., John Wiley \& Sons, Inc., New York (1985).

[DK] A. De Sole and V. Kac, Finite vs affine W-algebras, Jpn. J. Math. 1 (2006) 137-261.

[EK] A. G. Elashvili and V. G. Kac, Classification of good gradings of simple Lie algebras, Lie groups and invariant theory (E. B. Vinberg ed.), Amer. Math. Soc. Transl. 213 (2005) 85-104.

[F1] L. Fehér, L. O'Raifeartaigh, P. Ruelle, I. Tsutsui, and A. Wipf, Generalized Toda theories and $W$-algebras associated with integral gradings, Ann. Phys. 213 (1992) 1-20.

[F2] L. Fehér, L. O'Raifeartaigh, P. Ruelle, I. Tsutsui, and A. Wipf, On Hamiltonian reductions of the Wess-Zumino-Novikov-Witten theories, Phys. Rep. 222 (1992) 1-64.

[GG] W. L. Gan and V. Ginzburg, Quantization of Slodowy slices. Internat. Math. Res. Notices 5 (2002) 243-255.

[H] C.Hoyt, Good gradings of basic Lie superalgebras, Israel J. Math. 192 (2012) 251-280. 
[K] V. G. Kac, Lie superalgebras, Adv. Math. 26 (1977) 8-96.

[KW] V. G. Kac and M. Wakimoto, Integrable highest weight modules over affine superalgebras and number theory. Lie theory and geometry, 415456, Progr. Math., 123, Birkhäuser Boston, Boston, MA, 1994.

[Ko] B. Kostant, On Wittaker vectors and representation theory, Invent. Math. 48 (1978) 101-184.

[L1] I. Losev, Finite $W$-algebras, Proceedings of the International Congress of Mathematicians. Volume III, 1281-1307, Hindustan Book Agency, New Delhi, 2010. arXiv:1003.5811v1.

[L2] I. Losev, Quantized symplectic actions and W-algebras, J. Amer. Math. Soc. 23 (2010) 35-59.

[L3] I. Losev, Finite-dimensional representations of $W$-algebras, Duke Math. J. 159 (2011), no.1, 99-143.

[M] A. Molev, Yangians and classical Lie algebras, Mathematical Surveys and Monographs, 143, Amer. Math. Soc., Providence, RI, (2007).

[N] M. Nazarov, Yangian of the Queer Lie superalgebra, Commun. Math. Phys. 208 (1999) 195-223.

[NS] M. Nazarov, A. Sergeev, Centralizer construction of the Yangian of the queer Lie superalgebra, Studies in Lie Theory, 417-441, Progr. Math., 243 (2006).

[P] E. Poletaeva, On Kostant's Theorem for Lie superalgebras. In: Springer INdAM Series: Advances in Lie Superalgebras, P. Papi, M. Gorelik (eds). To be published.

[PS1] E. Poletaeva, V. Serganova, On finite W-algebras for Lie superalgebras in the regular case, In: "Lie Theory and Its Applications in Physics", V. Dobrev (ed), IX International Workshop. 20-26 June 2011, Varna, Bulgaria. Springer Proceedings in Mathematics and Statistics, Vol. 36 (2013) 487-497.

[PS2] E. Poletaeva, V. Serganova, On Kostant's theorem for the Lie superalgebra $Q(n)$, in preparation.

[Pr1] A. Premet, Special transverse slices and their enveloping algebras, Adv. Math. 170 (2002) 1-55.

[Pr2] A. Premet, Enveloping algebras of Slodowy slices and the Joseph ideal, J. Eur. Math. Soc. 9 (2007) 487-543.

[Pr3] A. Premet, Primitive ideals, non-restricted representations and finite $W$-algebras, Mosc. Math. J. 7 (2007) 743-762.

[S] A. Sergeev, The centre of envelloping algebra for Lie superalgebra $Q(n, \mathbb{C})$, Letters in Math. Phys. 7 (1983) 177-179.

[Sch] M. Scheunert, The Theory of Lie Superalgebras. In: Lecture Notes in Mathematics, 716, Springer, Berlin (1979).

[W] W. Wang, Nilpotent orbits and finite $W$-algebras, Geometric representation theory and extended affine Lie algebras, Fields Inst. Commun. 59, Amer. Math. Soc., Providence, RI, (2011), 71-105; arXiv:0912.0689v2. 
[Z] L. Zhao, Finite $W$-superalgebras for queer Lie superalgebras. arXiv:1012.2326v2. 